\title{
Mapas y clasificación de vegetación en ecosistemas estacionales: un análisis cuantitativo de los bosques secos de Piura
}

\author{
Vegetation maps and classification in seasonal ecosystems: a quantitative \\ analysis of the Piura dry forests
}

\author{
María de los Ángeles La Torre-Cuadros ${ }^{1}$ y Reynaldo Linares-Palomino ${ }^{2}$
}

\begin{abstract}
1 Herbario Forestal MOL, Universidad Nacional Agraria La Molina. Apdo. 456. Lima 1-Perú; Tel-Fax: +51-1-3493902, Tel: +511-4403342; Av. 8 de Octubre 184, 1-4403342; Av. 8 de Octubre 184,
Letra C, Miraflores, Lima 18-Perú. E-mail:

angeleslatorre@lamolina.edu.pe

2 Department of Systematic Botany, University of Goettingen, Untere Karspuele 2, 37073 Goettingen, Germany, E-mail: r.linaresp@yahoo.co.uk
\end{abstract}

\begin{abstract}
Resumen
Estudiamos datos de inventarios florísticos de 65 parcelas de 0,1 hectáreas de la vegetación estacional de las llanuras costeras de Piura usando métodos multivariados y análisis de composición florística. Identificamos seis grupos de vegetación, de los cuales cinco coinciden con tipos de vegetación descritos hace casi 100 años por Augusto Weberbauer. Por el contrario, de las ocho categorías de bosque seco definidas recientemente por el Proyecto Algarrobo para el área de estudio, ninguna pudo ser reconocida adecuadamente mediante métodos numéricos. Concluimos que los mapas y datos generados por Weberbauer resultan ser más exactos y revelan mejor las relaciones florísticas de la vegetación estacionalmente seca de Piura. Por lo tanto, sugerimos el uso más frecuente de las descripciones propuestas por Weberbauer para la región, paralelamente a los del Proyecto Algarrobo.
\end{abstract}

Palabras clave: Augusto Weberbauer, bosques estacionalmente secos, inventario, formaciones vegetales

\section{Abstract}

We studied data from 65 0.1-hectare floristic inventories from the seasonal lowland vegetation in coastal Piura by using multivariate methods and analyses of floristic composition. We identified six vegetation groups, five of which greatly coincide with vegetation types already described 100 years ago by August Weberbauer. In contrast, we were not able to accurately identify any of the eight dry forest categories recently defined by the Proyecto Algarrobo for the study area using numerical methods. We conclude that the maps and data generated by Weberbauer are more accurate and better reveal the floristic relationships of the seasonally dry vegetation in Piura. Therefore, we suggest a more widespread use of the descriptions made by Weberbauer in the region, parallel to those of the Proyecto Algarrobo.

Keywords: August Weberbauer, inventory, seasonally dry forest, vegetation formations

\section{Introducción}

Las clasificaciones de vegetación de escala regional son una herramienta valiosa para reforzar estrategias de conservación y manejo de bosques. Es en especial en las regiones tropicales, ricas en especies y endemismos, donde se necesitan con más urgencia ya que éstas enfrentan procesos acelerados de fragmentación y desaparición de la vegetación natural (Whitmore, 1997). Hay diversas formas de clasificar la vegetación. Whittaker (1973) reconoce doce enfoques diferentes, desde aquellos basados en unidades fisonómicas (Beard, 1955) y ambientales (Holdridge, 1947), hasta aquellos que usan unidades florísticas (Braun-Blanquet, 1964). Variaciones y combinaciones de estos enfoques han sido usados exitosamente en el trópico. Los bosques tropicales lluviosos de la Amazonia, por ejemplo, se han clasificado usando precipitación anual, combinaciones de condiciones de drenaje, topografía y estructura de vegetación o interpretación de imágenes satélite (Salovaara et al., 2004). La opción de clasificar detalladamente la vegetación basada en criterios puramente florísticos es muchas veces inviable por la cantidad de tiempo que se necesita invertir. Es por ello que existen numerosas experiencias de mapeo de vegetación que han combinado imágenes satélite e inventarios de diferente resolución e intensidad, respectivamente (Herbario Loja, 2001; Kuentz et al., 2007).

La vegetación más característica de la región del noroeste peruano, en especial en los departamentos de Tumbes, Piura y Lambayeque, es la de los bosques estacionalmente secos (Ferreyra, 1983; Linares-Palomino, 2004b). Esta región está fuertemente influenciada por eventos ENSO (El Niño-Southern Oscillation), que son un factor importante en los procesos de regeneración de los bosques estacionalmente secos (Holmgren et al., 2006a, b; Richter \& Ise, 2005). A pesar de que los bosques estacionalmente secos han sido considerados por algunos autores como bosques degradados, con baja diversidad biológica y estructural (ver Gentry, 1995), se ha demostrando que poseen un alto valor biológico (Aguirre-Mendoza et al., 2006; Sánchez et al., 2006) y cultural, ya que han sido fuente de vida para diversas culturas y grupos humanos (p.ej. Sicán) por cerca de 10000 años (Hocquenghem, 1999).

Las clasificaciones de los bosques estacionalmente secos del noroeste peruano también han sido sujetos de las diversas metodologías mencionadas líneas arriba (INRENA, 1975, 1995; Ferreyra, 1957; Sagástegui, 1989; Galán de Mera et al., 1997; ver resumen en Linares-Palomino, 2004a). La propuesta más reciente es del Proyecto Algarrobo, que ha producido mapas de cobertura forestal para los departamentos de Lambayeque, Piura y Tumbes (p.ej. INRENA, 2003). Estos mapas, producidos usando imágenes satélite Landsat 7 ETM+, corregidos y modificados de acuerdo con observaciones de campo, identifican y clasifican diferentes tipos de vegetación, presentando un nivel de detalle muy alto y por lo tanto se perfilarían como los más adecuados para estudios biológicos. Tenemos que resaltar en esta parte también la obra de Augusto Weberbauer, quien entre los años 1901-1905 y 1908-1940 recorrió casi todo el territorio peruano, entregándonos dos productos importantísimos: (1) una obra florística-fitogeográfica (Weberbauer, 1922, 1945) y (2) un mapa de vegetación (Weberbauer, 1922, 1930). En dicho mapa, Weberbauer clasifica la vegetación del Perú en 25 tipos. De estos, la vegetación seca de la región noroeste (sin considerar aquella de las vertientes húmedas occidentales) está compuesta por siete tipos, incluyendo los desiertos y semidesiertos.

A pesar de los varios estudios de vegetación en los bosques estacionalmente secos de la costa norte peruana, no se han 
Tabla 1. Tipos de vegetación en el departamento de Piura (INRENA, 2003) y número de parcelas muestreadas.

\begin{tabular}{|c|c|c|c|c|}
\hline Tipo de vegetación & $\begin{array}{l}\text { Sím- } \\
\text { bolo }\end{array}$ & $\begin{array}{l}\text { Área } \\
\text { (ha) }\end{array}$ & $\%$ & 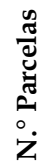 \\
\hline Algarrobal ribereño & $\mathrm{Ar}$ & 14320 & 0,4 & - \\
\hline Bs denso de colina & BsDC & 222065 & 6,2 & 8 \\
\hline Bs denso de llanura & BsDLl & 5884 & 0,1 & - \\
\hline Bs muy ralo de colina & BsmRC & 49542 & 1,4 & 1 \\
\hline Bs muy ralo de llanura & BsmRLl & 187287 & 5,2 & 5 \\
\hline Bs ralo de colina & BsRC & 40213 & 1,1 & 1 \\
\hline Bs ralo de llanura & BsRLl & 783893 & 21,9 & 19 \\
\hline Bs ralo de montaña & BsRM & 31614 & 0,9 & - \\
\hline Bs semi-denso de colina & BssDC & 332348 & 9,3 & 18 \\
\hline Bs semi-denso de llanura & BssDLl & 257661 & 7,2 & 6 \\
\hline Bs semi-denso de montaña & BssDM & 169437 & 4,7 & - \\
\hline Manglar & $\mathrm{Mg}$ & 636 & 0,02 & - \\
\hline Matorral & $\mathrm{Ma}$ & 304806 & 8,5 & 7 \\
\hline Matorral de dunas & Mad & 145988 & 4,1 & - \\
\hline Total & & 2545699 & & 65 \\
\hline
\end{tabular}

agotado aun las posibilidades de contribuir a su mejor conocimiento. Es por ello, que pretendemos con este artículo aportar a la clasificación de los bosques estacionalmente secos de la costa norte del Perú en general, y del departamento de Piura en particular. Nuestro objetivo principal es resaltar la importancia de los inventarios botánicos cuantitativos de la flora leñosa de los bosques estacionalmente secos y de su análisis mediante métodos numéricos, para revelar relaciones florísticas, así como comparar nuestros resultados con la clasificación del Proyecto Algarrobo (PA).

\section{Material y métodos \\ Área de estudio}

El departamento de Piura se localiza en el noroeste Peruano $\left(4^{\circ} 04^{\prime}-6^{\circ} 22^{\prime} \mathrm{S}, 79^{\circ} 12^{\prime}-81^{\circ} 19^{\prime} \mathrm{W}\right)$ y su vegetación más característica son los bosques secos. El mapa del Proyecto Algarrobo reconoce 14 tipos de vegetación presentes en las provincias de Talara, Sullana, Sechura, Paita, Piura, Morropón y Ayabaca (Tabla 1; INRENA, 2003). La topografía del departamento de Piura varía desde suave, cercana a las llanuras costeras en el oeste que alcanzan extensiones de $150 \mathrm{~km}$ antes de que comiencen las primeras estribaciones de los Andes, hasta montañas que alcanzan los $4000 \mathrm{~m}$ de altitud en el este. Restringimos el área de estudio al rango entre $37 \mathrm{~m}$ bajo nivel del mar en la Depresión de Bayovar hasta los $1600 \mathrm{~m}$ tierra adentro, donde se encuentran los tipos de bosque seco que crecen a mayores altitudes. El clima es árido a semi-árido en las llanuras costeras y en las faldas de los Andes. La precipitación es estacional y escasa, con alta variación interanual. Durante los eventos ENSO ocurren lluvias intensas e inundaciones (Ortlieb y Macharé, 1993).

\section{Muestreo y toma de datos}

Colectamos información de 57 parcelas de 0,1 ha $(20 \mathrm{~m} \mathrm{x}$ $50 \mathrm{~m}$ ), todas georeferenciadas y comprendidas entre el nivel del mar y aproximadamente $450 \mathrm{~m}$ de altitud. Las parcelas fueron distribuidas en número y en espacio según el área de cada tipo de bosque en el mapa del Proyecto Algarrobo y de acuerdo con la logística en campo. Adicionalmente, incluimos datos de ocho parcelas de 0,1 ha (10 transectos de $2 \times 50 \mathrm{~m}$ ) a altitudes mayores $\left(400 \_750 \mathrm{~m}\right)$ basados en Serván-Mori y Angulo-Pratolongo (2006).

La estacionalidad extremada de la precipitación en el área nos obligó a muestrear un área tan extensa como el departamento de Piura sólo durante la época seca, cuando las vías de acceso están en estado transitable y el ingreso a todo lugar está prácticamente asegurado. Esta ventaja contrasta sin embargo con la cantidad relativamente alta de especies que sólo se pueden colectar en estado infértil, esto es especialmente un problema en sub-arbustos y hierbas. Por el contrario, la identificación de especies leñosas arbustivas y arbóreas es factible aun en estado infértil. Por ello, en este trabajo decidimos colectar y registrar sólo especies leñosas arbustivas y arbóreas. Los datos de abundancia (número de individuos) de cada especie se obtuvieron registrando todas las plantas leñosas dentro de cada parcela. Los ejemplares colectados fueron identificados hasta especie o género cuando fue posible. De lo contrario, y para facilitar el análisis numérico, asignamos los ejemplares no identificados a grupos morfoespecíficos distintos cuando las características morfológicas y estructurales los diferenciaban de otros taxones ya identificados. Todos los ejemplares están depositados en el Herbario Forestal MOL de la Universidad Nacional Agraria La Molina. La nomenclatura de las leñosas sigue a W3-Tropicos (www.tropicos.org) y a la lista para bosques secos de Linares-Palomino y Pennington (2007), disponible en http://rbg-web2.rbge.org.uk/dryforest/database. htm. Dado que no pudimos evaluar los bosques secos de montaña, los análisis y la discusión se basan en datos colectados por debajo de los $1000 \mathrm{~m}$.

\section{Análisis de datos}

Construimos una matriz de parcelas por especies con datos de abundancia (número de individuos) de la cual eliminamos todas las especies incompletamente identificadas, así como especies que sólo ocurren en una sola parcela. La matriz final incluía 65 parcelas y 60 especies. Aplicamos análisis multivariados a la matriz para explorar las afinidades florísticas entre las parcelas. Las abundancias de todas las especies fueron transformadas logarítmicamente $[\log (\mathrm{x}+1)]$ para compensar el efecto de especies dominantes y menos numerosas o raras en los resultados estadísticos. La matriz transformada fue sometida a un análisis de clasificación UPGMA (Unweighted Pair Group Method with Arithmetic mean) y de ordenación de Escalamiento Multidimensional (MDS), usando el índice de disimilitud Bray-Curtis (o Sørensen cuantitativo) como medida de distancia en el MDS. Seleccionamos estos métodos porque son muy robustos para resumir, revelar y sugerir patrones en datos de ecología de comunidades (McCune y Grace, 2002).

Para comparar nuestros resultados con la clasificación del Proyecto Algarrobo, asignamos a cada una de nuestras parcelas en el MDS resultante el tipo de bosque que le corresponde según el mapa actual (INRENA, 2003). Evaluamos ambas clasificaciones (1) visualmente, comparando los patrones en la ordenación, y (2) mediante un Análisis Canónico de Coordenadas Principales -CAP- (Anderson y Robinson, 2003; Anderson y Willis, 2003) usando el índice de disimilitud Bray-Curtis como medida de dis- 
tancia. El Análisis Canónico de Coordenadas Principales permite incorporar hipótesis a priori tipo ANOVA en una ordenación canónica con la flexibilidad de poder utilizar cualquier tipo de distancia, y examinar tal hipótesis mediante un procedimiento por permutaciones.

El Proyecto Algarrobo define los diferentes tipos de bosque seco usando principalmente dos criterios: densidad (abundancia) de árboles por unidad de área y elevación sobre el nivel del mar (Anexo 1). Para explorar las relaciones de cada uno de estos factores con los patrones observados en la ordenación de las parcelas y ver su utilidad para definir tipos de bosques, correlacionamos cada uno de estos factores con la ordenación MDS. Para ello usamos la función vector fitting del paquete estadístico vegan, que permite ajustar vectores ambientales a las ordenaciones. Los vectores ajustados son representados gráficamente por flechas que indican la dirección del cambio más rápido en la variable ambiental (dirección del gradiente) y cuya longitud es proporcional a la correlación entre la ordenación y la variable ambiental (magnitud del gradiente) (Oksanen, 2006). Adicionalmente, también exploramos las relaciones de la ordenación MDS con la riqueza de especies, latitud y longitud (ambas en coordenadas UTM) de cada parcela. Los análisis multivariados y las correlaciones de factores ambientales fueron hechos en $R$ ( $R$ Development Core Team, 2006) utilizando el paquete vegan (Oksanen et al., 2006), PRIMER v5 (Clarke y Gorley, 2001) y CAP (Anderson, 2004).

\section{Resultados}

Registramos 92 especies y morfoespecies en las 6,5 ha inventariadas, totalizando 5880 individuos (la matriz que se usó para los análisis contenía 65 parcelas, 60 especies y 5428 individuos, ver Anexo 2). El número de especies por parcela fue desde una (parcela 56 donde Prosopis pallida (Humb. \& Bonpl. ex Willd.) Kunth era monodominante) a 41 (parcela 66). Hubo 22 especies que fueron registradas en una sola parcela, la mayoría de las cuales no pudo ser totalmente identificada. Las tres especies más frecuentes fueron Cordia lutea Lam. (registrada en 48 parcelas) y P. pallida y Loxopterygium huasango Spruce ex Engl. (ambas registradas en 35 parcelas). Las abundancias de especies por parcela fueron bastante variables $(90,4 \pm 84,7$ ind./parcela) y fueron máximas con 255 y 216 individuos para Croton cf. wagneri Müll. Arg. (parcela 49) e Indet.06 (parcela 54), respectivamente, esta ultima especie fue abundante solo en una parcela. Las abundancias por parcela estuvieron entre cuatro (parcela 3) y 325 individuos (parcela 54).

Obtuvimos resultados diferentes al comparar visualmente los productos de nuestro análisis con la clasificación del Proyecto Algarrobo. No pudimos encontrar muchas coincidencias. Después de asignar a cada una de nuestras parcelas, ordenadas según el resultado del MDS, la categoría de bosque seco que le correspondía según el mapa del Proyecto Algarrobo, pudimos observar varias categorías que se traslapaban (Fig. 1a). Esto lo pudimos advertir sobretodo en la categoría de bosque seco ralo de llanura (BsRLl) que formaba grupos conjuntamente con los matorrales (Ma), bosques secos muy ralos de llanura (BsmRLl), bosques secos semi-densos de colina (BssDC) y bosques secos ralos de colina (BsRC), entre otros.

Del análisis de clasificación y MDS reconocimos al menos seis grupos distintos de ensamblajes de especies de árboles (Fig. $1 b, 2)$ con un contenido entre tres a 25 parcelas:

A. Un bosque estacionalmente seco que crece por debajo de los $100 \mathrm{~m}$ y esta distribuido a lo largo de las planicies costeras del sur del departamento de Piura (Provincias de Sechura, Piura y Paita). Esta compuesto principalmente por las siempreverdes P. pallida y Capparis scabrida Kunth, y en menor medida por Capparis aviceniifolia Kunth, todas arbustivas, aunque en ocasiones llegan a formar pequeños árboles. La flora acompańante esta compuesta por Parkinsonia praecox (Ruiz \& Pav.) Hawkins, Galvezia fruticosa Gmel., Encelia canescens Lam. y Acacia macracantha Humb. \& Bonpl. ex Willd.

B. Un bosque estacionalmente seco que crece entre los 100 y $400 \mathrm{~m}$ y compuesto principalmente por $P$. pallida, $C$. lutea y $A$. macracantha. Esta formación, que se encuentra en las faldas inferiores del sur del departamento de Piura (Provincias de Piura, Sullana y Talara, principalmente), se acerca al litoral al norte de Talara debido a la influencia
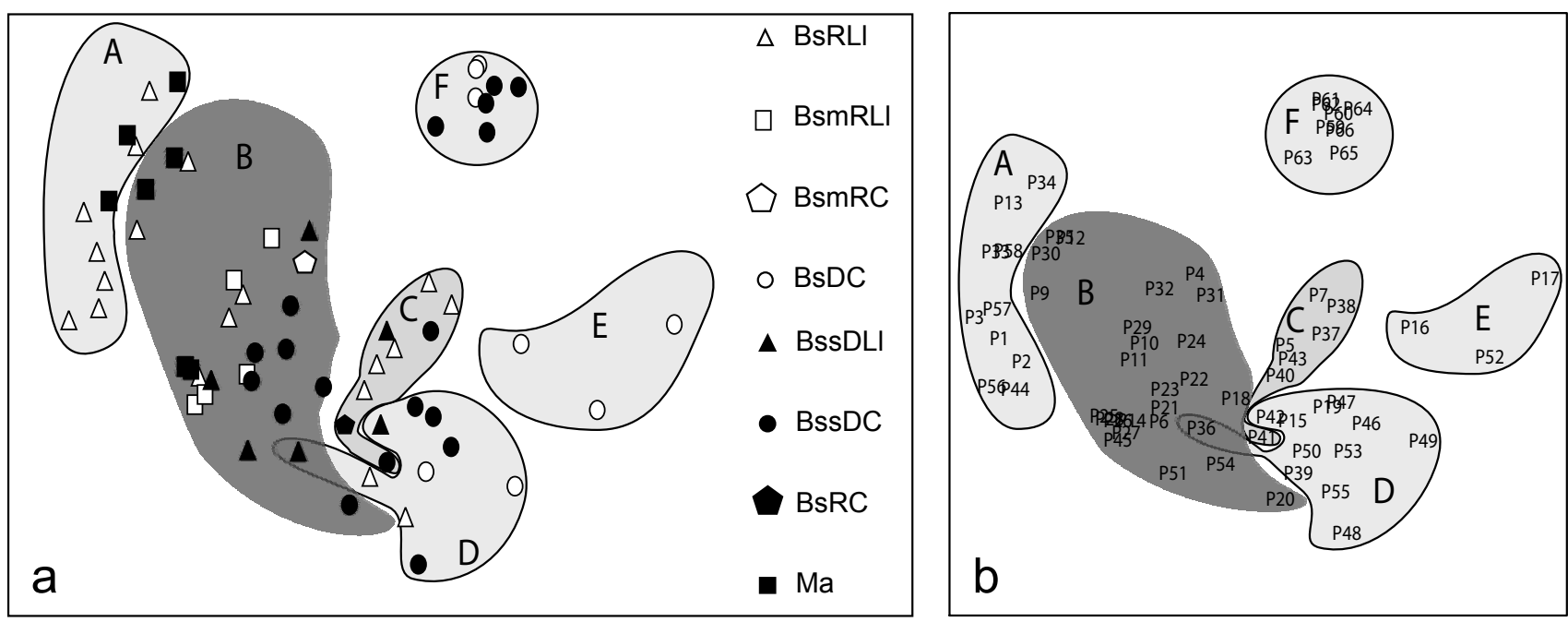

Figura 1. a) Ordenación MDS (usando índice de disimilitud Bray-Curtis) de las 65 parcelas con datos transformados logarítmicamente (log[x+1]), estrés: 0,15; reiteraciones: 9999). Se muestra cada parcela codificada según el tipo de bosque del mapa del Proyecto Algarrobo (PA) (las abreviaturas de cada tipo de bosque se pueden extraer de la Tabla 1). (b) La misma ordenación MDS anterior con los seis grupos de bosque seco obtenidos en el UPGMA. Nótese el solapamiento entre los grupos B y D. 


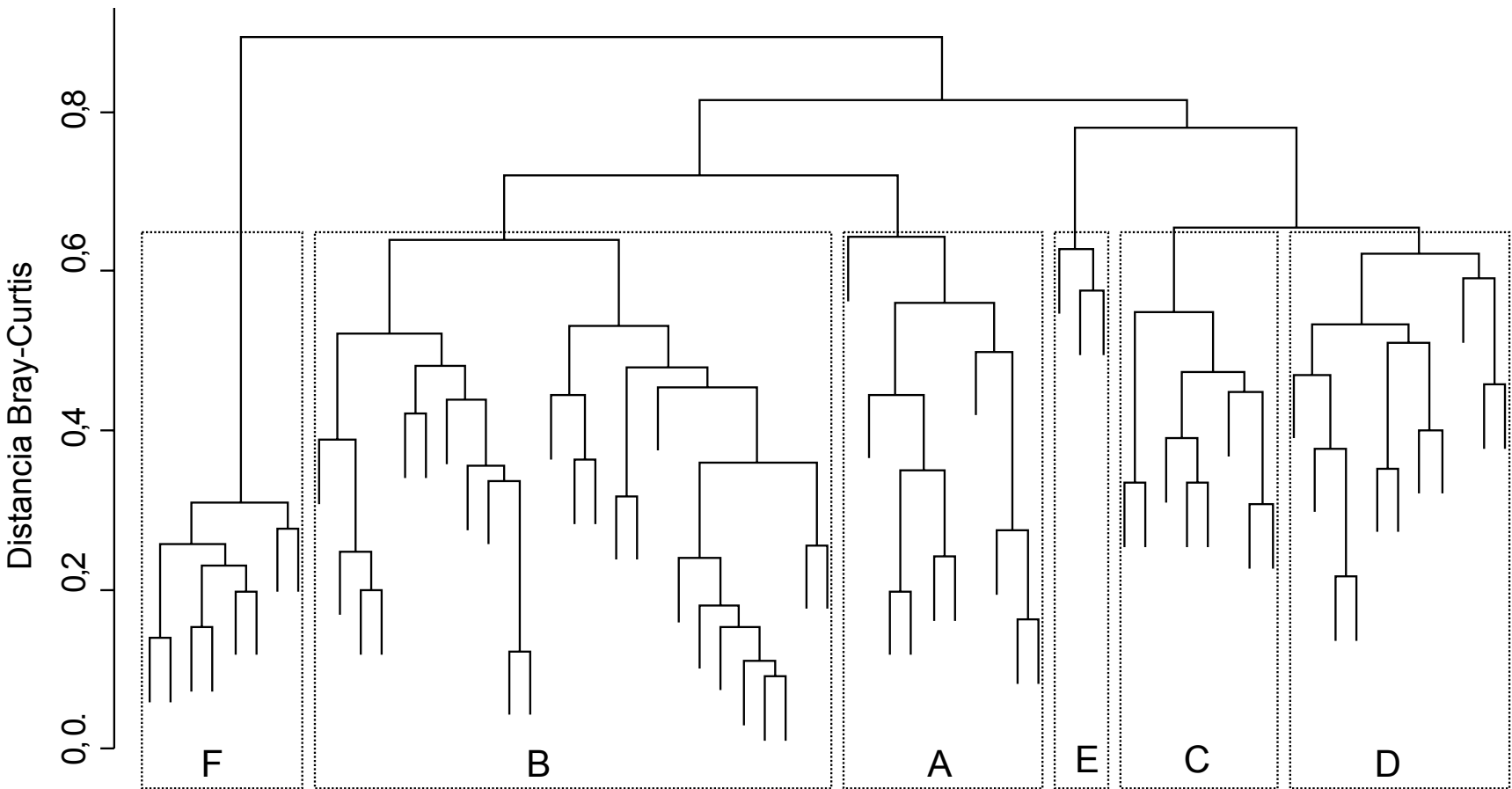

Figura 2. Dendrograma resultante del análisis UPGMA (usando índice de disimilitud Bray-Curtis), mostrando los seis grupos de bosque seco mencionados en el texto.

orográfica de la Cordillera de los Amotapes y la presencia de los tablazos (Máncora y Talara). Estas características favorecen la presencia de lluvias más intensas y también la de especies con requerimientos hídricos más exigentes (p.ej. B. graveolens). La flora acompañante conspicua está compuesta por Armatocereus cartwrightianus (Britton \& Rose) Backeb. ex A.W. Hill, Caesalpinia glabrata Kunth, C. avicenniifolia, $C$. scabrida, Loxopterygium huasango Spruce ex Engl., Neoraimondia arequipensis (Meyen) Backeb., P. praecox. Además hemos encontrado presencia importante de arbustos de E. canescens y pequeños árboles de G. ulmifolia Lam.

C. Un bosque estacionalmente seco que crece entre los 200 y $350 \mathrm{~m}$ y compuesto principalmente por Bursera graveolens (Kunth) Triana \& Planch., C. scabrida, C. lutea y G. ulmifolia.
Esta formación se encuentra restringida a un área al sur de Piura, en la provincia de Morropón.

D. Un bosque estacionalmente seco que crece entre los 220 y $470 \mathrm{~m}$ y compuesto principalmente por Coccoloba ruiziana Lindau, C. glabrata, L. huasango, C. lutea e Ipomoea carnea Jacq. Estos bosques constituyen la franja inferior de los bosques estacionalmente secos de colina y montaña y se distribuyen de norte a sur del departamento de Piura, siguiendo la dirección de la cordillera, en las Provincias de Sullana, Ayabaca y Morropón.

E. Un bosque estacionalmente seco que crece entre los 280 y 350 m y compuesto principalmente por Alseis peruviana Standl., Simira rubescens (Benth.) Bremekamp ex Steyermark, pero

Tabla 2. Validación cruzada (cross-validation) obtenida por el método CAP con los tipos de bosques propuestos por el Proyecto Algarrobo (PA) (6 grupos, total correcto: $24 / 63=38,095 \%$, error de clasificación errónea: $61,905 \%$. BsmRC y BsRc no se incluyen por tener una parcela cada uno) y los grupos obtenidos con UPGMA (6 grupos; total correcto: $61 / 65=93,846 \%$, error de clasificación errónea: $6,154 \%$ ). NTP: número total de parcelas (las abreviaturas de cada tipo de bosque se pueden extraer de la Tabla 1 y texto).

\begin{tabular}{llllllllc}
\hline Grupos PA & BsDC & BsmRLI & BsRLI & BssDC & BssDLI & Ma & NTP & \% Correcto \\
\hline BsDC & 7 & 0 & 0 & 1 & 0 & 0 & 8 & 87,50 \\
BsmRLI & 0 & 3 & 0 & 0 & 2 & 0 & 5 & 60,00 \\
BsRLI & 0 & 3 & 0 & 4 & 3 & 9 & 19 & 0,00 \\
BssDC & 5 & 2 & 0 & 8 & 3 & 0 & 18 & 44,44 \\
BssDLI & 0 & 2 & 1 & 1 & 2 & 0 & 6 & 33,33 \\
Ma & 0 & 3 & 0 & 0 & 0 & 4 & 7 & 57,14 \\
\hline Grupos UPGMA & A & B & C & D & E & F & NTP & $\%$ Correcto \\
\hline A & 10 & 0 & 0 & 0 & 0 & 0 & 10 & 100,00 \\
B & 0 & 23 & 0 & 2 & 0 & 0 & 25 & 92,00 \\
C & 0 & 0 & 8 & 0 & 0 & 0 & 8 & 100,00 \\
D & 0 & 0 & 0 & 10 & 1 & 0 & 11 & 90,91 \\
E & 0 & 0 & 0 & 1 & 2 & 0 & 3 & 66,67 \\
F & 0 & 0 & 0 & 0 & 0 & 8 & 8 & 100,00 \\
\hline
\end{tabular}


también con elementos conspicuos de Terminalia valverdae A.H. Gentry, L. huasango, Piscidia carthagenensis Jacq., Pithecellobium excelsum (Kunth) Benth., Eriotheca ruizii (K. Schum.) A. Robyns. Esta formación se encuentra distribuida en el extremo norte de la provincia de Sullana.

F. Un bosque estacionalmente seco que crece entre los 390 y 750 $\mathrm{m}$ y compuesto principalmente por E. ruizii, que es la especie más conspicua y abundante, acompañada por $P$. excelsum y Erythrina smithiana Krukoff. Están presentes conspicuamente también Maytenus sp.1, Celtis iguanaea (Jacq.) Sarg., Leucaena trichodes (Jacq.) Benth., Albizia multiflora (Kunth) Barneby \& J.W. Grimes, A. macracantha, L. huasango y B. graveolens. Esta formación es característica de las faldas y laderas occidentales de los Andes del extremo sur de la provincia de Huancabamba.

Por su parte, el análisis CAP nos mostró que los seis tipos de bosque identificados con métodos numéricos (UPGMA y MDS) resultaron ser más confiables que los presentados en el mapa del Proyecto Algarrobo. Los grupos formados por UPGMA tienen un 93,846\% de corresponder a una clasificación correcta mientras que la clasificación de las parcelas codificadas según las categorías del mapa del Proyecto Algarrobo fue sólo correcta en un $38,095 \%$ (Tabla 2).

Finalmente, encontramos correlaciones positivas y significantes entre la ordenación del MDS y (en orden decreciente) riqueza de especies $(r=0,85, p<0,001)$, altitud $(r=0,76, p<0,001)$, longitud $(\mathrm{r}=0,64, \mathrm{p}<0,001)$, latitud $(\mathrm{r}=0,59, \mathrm{p}<0,001)$ y abundancia por parcela $(r=0,55, \mathrm{p}<0,001)$ (todos los valores $\mathrm{p}$ basados en 1000 permutaciones).

\section{Discusión}

De las seis formaciones encontradas, pudimos reconocer a cinco en los trabajos de Augusto Weberbauer. La Formación A fue reconocida de manera idéntica por Weberbauer $(1922,1930)$ en su carta de vegetación de los Andes peruanos como "leñosas muy dispersas, siempreverdes, mantenidas por el agua subterránea". La Formación B, es similar a la que Weberbauer (1930) definió como "vegetación xerofítica, compuesta a manera de sabana, donde los árboles, arbustos y cactáceas se hallan dispersos sobre un suelo cubierto de pequeñas gramíneas". Weberbauer $(1922,1930)$ reconoció a la Formación C como "leñosas dispersas, siempreverdes, mantenidas por el agua subterránea”, muy similar a la primera, pero con algunas especies caducifolias adicionales y presencia de algunas herbáceas. La Formación D es parte de la "vegetación xerofítica, compuesta a manera de parque, con árboles, arbustos, cactáceas y herbazales" de Weberbauer (1930). La Formación E no la hemos podido ubicar adecuadamente dentro del esquema de Weberbauer, pero posiblemente sea parte o quizás una transición entre la "vegetación xerofítica, compuesta a manera de parque" y la muy similar de "montes macrotérmicos, compuestos de árboles, arbustos y cactáceas". Weberbauer (1930) reconoció a la Formación F como "montes macrotérmicos, compuestos de árboles, arbustos y cactáceas, donde, terminada la estación lluviosa, la mayor parte de los árboles y arbustos se deshojan”. Podemos ver que lo tipos de vegetación definidos por Weberbauer (1922, incluyendo la adición de 1930), a pesar de haber sido publicados hace casi 100 ańos, pueden todavía ser reconocidos hoy en día. Un ejercicio interesante sería ubicar nuestras parcelas en los mapas de vegetación de Weberbauer y examinar si cada una de ellas no sólo coincide en su composición sino también en su ubicación con la propuesta de Weberbauer. Lamentablemente, Weberbauer no pudo actualizar su mapa de vegetación como lo hizo con su libro. A la versión de 1922 sólo pudo agregarle una extensión que comprendía el norte de Piura y Tumbes en 1930, resultado de sus exploraciones botánicas por esa región en 1927. Ambos mapas no son totalmente complementarios y usan, en algunos casos, terminología diferente (sobre todo si se compara las definiciones de sus cartas de 1922 y del libro de 1945), probablemente como resultado de la evolución de sus ideas respecto de la vegetación en esta región, dificultando el análisis de todos nuestros datos de manera conjunta. Sin embargo, analizando cada carta por separado, notamos que muchas de las parcelas correspondían con los diferentes tipos de bosque.

Si consideramos los grupos encontrados en este trabajo con métodos numéricos, observamos que ninguno está compuesto por un único tipo de bosque de la propuesta del Proyecto Algarrobo, excepto el grupo E, compuesto por parcelas que fueron identificadas como bosque seco denso de colina. Sin embargo, parcelas con esta misma clasificación se encuentran formando parte de los grupos D y F. Situaciones similares fueron evidentes en las ordenaciones basadas sólo en las clases de altura y densidad propuestas por el Proyecto Algarrobo (datos no mostrados). Por ejemplo, no se pudo observar una separación clara entre las parcelas por debajo y encima de los $250 \mathrm{~m}$ de altitud. Soporte a estas afirmaciones las obtuvimos del análisis objetivo de ambos sistemas de clasificación mediante el método CAP, este fue bastante útil para evaluar la reclasificación de las muestras en cada tipo de bosque. Todos los factores que hemos analizado en cuanto a su relación con el tipo de bosque han resultado en correlaciones altamente significativas. Si bien existe una correlación entre la altitud y, en menor medida, la densidad de especies con el tipo de bosque (tal como lo aplicó el Proyecto Algarrobo), no son los únicos factores que deben ser considerados. Información fácil de medir durante un inventario, como la altitud, latitud, longitud y en especial riqueza y composición de especies, son igualmente importantes y necesarios. Información adicional que recomendamos obtener durante inventarios, pero que generalmente implican incrementos en los costos y en el tiempo, son análisis de suelos.

El mapa del Proyecto Algarrobo ha sido una contribución interesante para el avance del conocimiento de los tipos de vegetación en Piura. Sin embargo, basados en los resultados de este trabajo, creemos que puede conducir a conclusiones erradas respecto a características ecológicas o florísticas de la vegetación, ya que, al menos para el departamento de Piura, no parece mostrar adecuadamente los tipos de vegetación. Por el contrario, es destacable la vigencia de la clasificación de vegetación de Weberbauer para la región, por lo que proponemos que se utilice su mapa y la nomenclatura correspondiente con más regularidad de lo que se viene haciendo. El paso lógico siguiente, es el análisis numérico de un mayor conjunto de datos florísticos cuantitativos de la vegetación estacional en Piura, con el fin de afinar, afirmar y quizás, en algunos casos, modificar las formaciones de vegetación que propuso A. Weberbauer. Este trabajo debe lógicamente extenderse a los departamentos adyacentes de Lambayeque y 
Tumbes, cuyos mapas de bosques secos fueron producidos bajo los mismos criterios que el de Piura y podrían tener deficiencias similares a las expuestas en este trabajo.

\section{Agradecimientos}

El presente trabajo se enriqueció mucho con comentarios y sugerencias de A. Galán de Mera, M. Holmgren y S. HerrándoPerez. El equipo de trabajo de la División de Manejo de Recursos Naturales del Centro Internacional de la Papa apoyó logísticamente y proporcionó el financiamiento. S. Montero, T. Álvarez y L. Criollo asistieron en el trabajo de campo. C. Reynel, S. Novoa y A. Galán de Mera absolvieron dudas taxonómicas en la determinación de algunas especies. F. Angulo-Pratolongo y A. Serván-Mori proporcionaron gentilmente los datos de sus parcelas para ser incluidos en este trabajo. Finalmente, nuestro agradecimiento a las autoridades y pobladores de las provincias de Talara, Sullana, Sechura, Paita y Piura que en varias ocasiones proporcionaron hospedaje en sus centros comunales ó casas, colaborando con información indispensable para facilitar las diversas actividades de campo.

\section{Literatura citada}

Aguirre-Mendoza, Z., R. Linares-Palomino \& L.P. Kvist. 2006. Especies leñosas y formaciones vegetales en los bosques estacionalmente secos de Ecuador y Perú. Arnaldoa 13: 324-350.

Anderson, M.J. 2004. CAP: a FORTRAN computer program for canonical analysis of principal coordinates. Department of Statistics. University of Auckland, New Zeland.

Anderson, M.J. \& J. Robinson. 2003. Generalized discriminant analysis based on distances. Australian and New Zealand Journal of Statistics 45: 301-318.

Anderson, M.J. \& T.J. Willis. 2003. Canonical analysis of principal coordinates: a useful method of constrained ordination for ecology. Ecology 84: 511-525.

Beard, J.S. 1955. The classification of tropical American vegetationtypes. Ecology 36: 89-100.

Braun-Blanquet, J. 1964. Pflanzensoziologie 3 ed. Berlin, Wien, New York: Springer.

Clarke, K.R. \& R.N. Gorley. 2001. PRIMER v5. User Manual/Tutorial. Primer-E Ltd., Plymouth.

Ferreyra, R. 1957. Contribución al conocimiento de la flora costanera del norte Peruano (Departamento de Tumbes). Boletín de la Sociedad Argentina de Botánica 6:194-206.

Ferreyra, R. 1983. Los tipos de vegetación de la costa peruana. Anales del Jardín Botánico de Madrid 40: 241-256.

Galán De Mera, A., J.A. Vicente Orellana, J.A. Lucas García \& A. Probanza Lobo. 1997. Phytogeographical sectoring of the Peruvian coast. Global Ecology and Biogeography Letters 6: 349-367.

Gentry, A.H. 1995. Diversity and floristic composition of neotropical dry forests. En: S.H. Bullock, H.A. Mooney y E. Medina, eds. Seasonally dry tropical forests. Cambridge University Press, Cambridge, U.K. Pp. 146-194.

Herbario LOJA. 2001. Zonificación y determinación de los tipos de vegetación del bosque seco en el sur-occidente de la provincia de Loja. Loja, Ecuador.

Hocquenghem, A.M. 1999. Para vencer la muerte. Piura y Tumbes. Raíces en el bosque seco y en la selva alta-Horizontes en el Pacífico y en la Amazonia. CNRS/IFEA/INCAH, Lima.

Holdridge, L.R. 1947. Determination of world plant formations from simple climatic data. Science 105: 367-368.
Holmgren M., B.C. López, J.R. Gutiérrez \& F.A. Squeo. 2006a. Herbivory and plant growth rate determine the success of El Niño Southern Oscillation-driven tree establishment in semiarid South America. Global Change Biology 12: 2263-2271.

Holmgren M., P. Stapp, C.R. Dickman, C. Gracia, S. Graham, J.R. Gutiérrez, C. Hice, F. Jaksic, D.A. Kelt, M. Letnic, M. Lima, B.C. López, P.L. Meserve, W.B. Milstead, G.A. Polis, M.A. Previtali, M. Richter, S. Sabaté y F.A. Squeo. 2006b. Extreme climatic events shape arid and semiarid ecosystems. Frontiers in Ecology and the Environment 4: 87-95.

INRENA (Instituto Nacional De Recursos Naturales). 1975. Mapa Ecológico del Perú escala 1:1000000 con guía explicativa. Lima, Perú.

INRENA (Instituto Nacional De Recursos Naturales). 1995. Mapa Forestal del Perú escala 1:1000000 con guía explicativa. Lima, Perú.

INRENA (Instituto Nacional De Recursos Naturales). 1998. Mapa de bosques secos del Departamento de Piura. Memoria descriptiva. INR-82-DGEP. Lima-Perú.

INRENA 2003. Mapa de los Bosques Secos del departamento de Piura. Memoria Descriptiva. INRENA Proyecto Algarrobo.

Kuentz, A., A. Galán de Mera, M.-P. Ledru \& J.-C. Thouret. 2007. Phytogeographical data and modern pollen rain of the puna belt in southern Peru (Nevado Coropuna, Western Cordillera). Journal of Biogeography 34: 1762-1776.

Linares-Palomino, R. 2004a. Los Bosques Tropicales Estacionalmente Secos: I. El concepto de los bosques secos en el Perú. Arnaldoa 11:85-102.

Linares-Palomino, R. 2004b. Los Bosques Tropicales Tropicales Estacionalmente Secos: II. Fitogeografía y Composición Florística. Arnaldoa 11: 103-138.

Linares-Palomino, R. \& R.T. Pennington. 2007. Lista anotada de plantas leñosas en bosques estacionalmente secos del Perú - una nueva herramienta en Internet para estudios taxonómicos, ecológicos y de biodiversidad. Arnaldoa 14: $149-152$

McCune, B. \& J.B. Grace. 2002. Analysis of Ecological Communities. MJM Press. Oregon.

Oksanen, J. 2006. (en línea) Multivariate Analysis of Ecological Communities in R: vegan tutorial. $<\mathrm{http}: / / \mathrm{cc}$.oulu. fi/ jarioksa/softhelp/vegan.html>. Acceso 17/11/2006.

Oksanen, J., R. Kindt, P. Legendre y R.B. O'Hara. 2006. Vegan: Community Ecology Package version 1.8-3. <http://cran. r-project.org/>. Acceso 17/11/2006.

Ortlieb, L. \& J. Macharé. 1993. Former El Niño events: records from western South America. Global and Planetary Change 7: 181-202.

Proyecto Algarrobo. 1993. Mapa e inventario forestal de los bosques secos de Lambayeque. Memoria explicativa. CEIMADProyecto Algarrobo, Chiclayo, Perú.

R Development Core Team. 2006. R: A language and environment for statistical computing. R Foundation for Statistical Computing, Vienna, Austria. ISBN 3-900051-07-0, URL http://www.R-project.org.

Richter, M. y M. Ise. 2005. Monitoring plant development after El Niño 1997/98 in Northwestern Perú. Erdkunde 59: 136-155.

Sagástegui, A. 1989. Vegetación y flora de la provincia de Contumazá. CONCYTEC, Trujillo.

Salovaara, K.J., G.G. Cárdenas y H. Tuomisto. 2004. Forest classification in an Amazonian rainforest landscape using pteridophytes as indicator species. Ecography 27: 689-700. 
Sánchez T., O., L.P. Kvist \& Z. Aguirre M. 2006. Bosques secos en Ecuador y sus plantas útiles. En: M. Moraes R., B. Øllgaard, L.P. Kvist, F. Borchsenius \& H. Balslev, eds. Botánica Económica de los Andes Centrales. Universidad Mayor de San Andrés, Plural Editores, La Paz. Pp. 188-204.

Serván-Mori, A. \& F. Angulo-Pratolongo. 2006. Caracterización Florística y Análisis de Diversidad en el área de distribución de la Pava Aliblanca (Penelope albipennis Taczanowski). Zonas Áridas 10: 84-101.

Weberbauer, A. 1922. Die Vegetationskarte der peruanischen Anden zwischen $5^{\circ}$ und $17^{\circ} \mathrm{S}$. Petermanns Geographische Mitteilungen, 68: 89-91.
Weberbauer, A. 1930. Die Pflanzendecke Nordperus im Departamento Tumbez una angrenzenden Teilen des Departamento Piura $\left(3^{\circ} 30^{4}-5^{\circ} \mathrm{s}\right.$. Br.). Botanische Jahrbücher 63: 29-48.

Weberbauer, A. 1936. Phytogeography of the Peruvian Andes. Field Museum of Natural History, Botanical Series 13:13-81.

Weberbauer, A. 1945. El mundo vegetal de los Andes peruanos. Ministerio de Agricultura, Dirección de Agricultura, Estación Experimental Agrícola de La Molina, Lima.Pp:776.

Whitmore, T.C. 1997. Tropical forest disturbance, disappearance, and species loss. En: W.F. Laurence and R.O. Bierregaard, Jr., eds. Tropical forest remnants. The University of Chicago Press, Chicago. Pp. 2-28.

Whittaker, R.H. 1973. Approaches to classifying vegetation. En: R.H. Whittaker, ed. Ordination and Classification of Communities. Handbook of Vegetation Science 5. Junk, The Hague. Pp. 323-354.

Anexo 1. Bosques secos evaluados en el presente trabajo (1-5) mostrando los dos criterios: densidad (abundancia) de árboles por unidad de área y elevación sobre el nivel del mar del Proyecto Algarrobo (PA) y los grupos propuestos según análisis numérico (7-12) con su respectivo rango altitudinal.

\begin{tabular}{lcc}
\hline Grupos & Densidad (ind./ha) & Altitud (m) \\
\hline 1. BsDC & $>150$ & $\geq 250<1000$ \\
2. BsmRLI & $\leq 25$ & $<250$ \\
3. BsRLI & $>25 \leq 70$ & $<250$ \\
4. BssDC & $>70 \leq 150$ & $\geq 250<1000$ \\
5. BssDLI & $>70 \leq 150$ & $<250$ \\
6. Ma & - & - \\
7. A & - & $<100$ \\
8. B & - & $100-400$ \\
9. C & - & $200-350$ \\
10. D & - & $220-470$ \\
11. E & - & $280-350$ \\
12. F & - & $390-750$ \\
\hline
\end{tabular}


Anexo 2. Matriz de abundancias usada para los análisis numéricos ( 65 parcelas, 60 especies y 5428 individuos).

\section{Especie}

Loxopterygium huasango Spruce ex Engl.

Tagetes sp.

Vernonia sp.

Encelia canescens Lam. var. canescens

Tecoma sp.

Cochlospermum vitifolium (Willd.) Spreng.

Cordia lutea Lam.

Bursera graveolens (H. B. K.) Triana \& Planch.

Opuntia sp.

Neoraimondia arequipensis (Meyen) Backeb.

Armatocereus cartwrightianus (Britton \& Rose) Backeb. ex A.W.Hill

Capparis sp. 2

Capparis crotonoides Kunth

Capparis mollis Kunth

Capparis avicenniifolia Kunth

Capparis scabrida Kunth

Maytenus sp.2

Maytenus sp.1

Ipomoea carnea Jacquin

Croton sp.

Croton cf. wagneri Müll. Arg.

Hyptis sp.

Senna undulata (Benth.) H.Irwin \& Barneby

Parkinsonia praecox (Ruiz \& Pav.) Hawkins subsp. praecox

Caesalpinia glabrata Kunth

Albizia multiflora (Kunth) Barneby \& J.W. Grimes

Mimosa cf. acantholoba (Humb. \& Bonpl. ex Willd.) Poir.

Leucaena trichodes (Jacq.) Benth.

Pithecellobium excelsum (Kunth) Mart.

Acacia macracantha H. \& B. ex Willdenow

Prosopis pallida (Humb. \& Bonpl. ex Willd.) Kunth

Piscidia carthagenensis Jacq.

Geoffroea spinosa Jacq.

Erythrina smithiana Krukoff

Buddleja americana $\mathrm{L}$.

Ceiba trichistandra (A. Gray) Bakh.

Eriotheca ruizii (K. Schum.) A. Robyns

Guazuma ulmifolia (Lam.) Kuntze var. ulmifolia

Muntingia calabura L.

Ficus nymphacifolia Mill.

Myrcianthes discolor (HBK) McVaugh

Bougainvillea peruviana Bonpl.

Bougainvillea pachyphylla Heimerl ex Standl.

Pisonia macranthocarpa J. D. Smith

Ximenia americana $\mathrm{L}$.

Priogymnanthus sp.

Piper sp.

Coccoloba densifrons Mart.

Coccoloba sp.

Coccoloba ruiziana Lindau

Scutia spicata (Humb. \& Bonpl. ex Willd.) Weberb. var. spicata

Alseis peruviana Standl.

Simira rubescens (Benth.) Bremek. ex Steyerm.

Sideroxylon sp.

Galvesia fruticosa J. F. Gmel.

Duranta sp.

Acnistus arborescens (L.) Schltdl.

Cestrum auriculatum L'Her.

Celtis iguanaea (Jacq.) Sarg.

Phoradendron sp.

\section{$\begin{array}{llllllllllllll}\text { P01 P02 P03 P04 } & \text { P05 } & \text { P06 } & \text { P07 } & \text { P09 } & \text { P10 } & \text { P11 } & \text { P12 } & \text { P13 } & \text { P14 } & \text { P15 }\end{array}$}

\begin{tabular}{|c|c|c|c|c|c|c|c|c|c|c|c|c|c|}
\hline 0 & 0 & 0 & 0 & 2 & 0 & 7 & 0 & 0 & 0 & 0 & 0 & 0 & 2 \\
\hline 0 & 0 & 0 & 2 & 2 & 0 & 0 & 0 & 0 & 0 & 0 & 0 & 0 & 0 \\
\hline 0 & 0 & 0 & 0 & 0 & 0 & 0 & 0 & 0 & 0 & 0 & 0 & 0 & 0 \\
\hline 0 & 0 & 0 & 0 & 0 & 0 & 0 & 14 & 0 & 0 & 0 & 0 & 0 & 0 \\
\hline 0 & 0 & 0 & 0 & 0 & 0 & 0 & 0 & 0 & 0 & 0 & 0 & 0 & 0 \\
\hline 0 & 0 & 0 & 0 & 0 & 0 & 0 & 0 & 0 & 0 & 0 & 0 & 0 & 0 \\
\hline 0 & 0 & 0 & 15 & 10 & 17 & 4 & 0 & 18 & 26 & 0 & 0 & 28 & 14 \\
\hline 0 & 0 & 0 & 1 & 1 & 0 & 0 & 0 & 0 & 0 & 0 & 0 & 0 & 1 \\
\hline 0 & 0 & 0 & 0 & 0 & 0 & 0 & 0 & 0 & 0 & 0 & 0 & 0 & 0 \\
\hline 0 & 0 & 0 & 0 & 0 & 0 & 0 & 0 & 0 & 0 & 0 & 0 & 0 & 0 \\
\hline 0 & 0 & 0 & 0 & 0 & 0 & 0 & 0 & 0 & 0 & 0 & 0 & 0 & 0 \\
\hline 0 & 0 & 0 & 1 & 3 & 0 & 5 & 0 & 0 & 0 & 0 & 0 & 0 & 0 \\
\hline 0 & 0 & 0 & 0 & 0 & 0 & 15 & 0 & 0 & 0 & 0 & 0 & 0 & 0 \\
\hline 0 & 0 & 0 & 0 & 0 & 0 & 2 & 0 & 0 & 0 & 0 & 0 & 0 & 0 \\
\hline 0 & 0 & 0 & 6 & 2 & 0 & 1 & 0 & 0 & 0 & 0 & 20 & 0 & 0 \\
\hline 1 & 1 & 0 & 0 & 2 & 1 & 15 & 1 & 2 & 0 & 3 & 2 & 0 & 1 \\
\hline 0 & 0 & 0 & 0 & 0 & 0 & 0 & 0 & $\overline{0}$ & 0 & 0 & $\overline{0}$ & 0 & 0 \\
\hline 0 & 0 & 0 & 0 & 0 & 0 & 0 & 0 & 0 & 0 & 0 & 0 & 0 & 0 \\
\hline 0 & 0 & 0 & 0 & 0 & 0 & 2 & 0 & 0 & 0 & 0 & 0 & 0 & 14 \\
\hline 0 & 0 & 0 & 0 & 0 & 0 & 0 & 0 & 0 & 0 & 0 & 0 & 0 & 0 \\
\hline 0 & 0 & 0 & 0 & 0 & 0 & 0 & 0 & 0 & 0 & 0 & 0 & 0 & 0 \\
\hline 0 & 0 & 0 & 0 & 0 & 0 & 0 & 0 & 0 & 0 & 0 & 0 & 0 & 0 \\
\hline 0 & 0 & 0 & 0 & 0 & 0 & 0 & 0 & 0 & 0 & 0 & 0 & 0 & 0 \\
\hline 0 & 0 & 0 & 1 & 0 & 1 & 0 & 0 & 0 & 0 & 0 & 0 & 0 & 0 \\
\hline 0 & 0 & 0 & 0 & 1 & 0 & 1 & 0 & 0 & 0 & 0 & 0 & 0 & 2 \\
\hline 0 & 0 & 0 & 0 & 0 & 0 & 0 & 0 & 0 & 0 & 0 & 0 & 0 & $\overline{0}$ \\
\hline 0 & 0 & 0 & 0 & 0 & 3 & 0 & 0 & 0 & 0 & 0 & 0 & 0 & 0 \\
\hline 0 & 0 & 0 & 0 & 0 & 0 & 0 & 0 & 0 & 0 & 0 & 0 & 0 & 0 \\
\hline 0 & 0 & 0 & 0 & 0 & 0 & 0 & 0 & 0 & 0 & 0 & 0 & 0 & 0 \\
\hline 0 & 0 & 1 & 20 & 0 & 0 & 0 & 10 & 15 & 22 & 34 & 0 & 0 & 0 \\
\hline 5 & 22 & 3 & 0 & 0 & 2 & 0 & 84 & 5 & 7 & 11 & 2 & 31 & 0 \\
\hline 0 & 0 & 0 & 0 & 0 & 0 & 0 & 0 & 0 & 0 & 0 & 0 & 0 & 0 \\
\hline 0 & 0 & 0 & 0 & 0 & 0 & 0 & 0 & 0 & 0 & 0 & 0 & 0 & 0 \\
\hline 0 & 0 & 0 & 0 & 0 & 0 & 0 & 0 & 0 & 0 & 0 & 0 & 0 & 0 \\
\hline 0 & 0 & 0 & 0 & 0 & 0 & 0 & 0 & 0 & 0 & 0 & 0 & 0 & 0 \\
\hline 0 & 0 & 0 & 0 & 0 & 0 & 0 & 0 & 0 & 0 & 0 & 0 & 0 & 0 \\
\hline 0 & 0 & 0 & 0 & 0 & 0 & 0 & 0 & 0 & 0 & 0 & 0 & 0 & 0 \\
\hline 0 & 0 & 0 & 0 & 19 & 0 & 6 & 0 & 0 & 0 & 0 & 0 & 0 & 0 \\
\hline 0 & 0 & 0 & 0 & 0 & 0 & 0 & 0 & 0 & 0 & 0 & 0 & 0 & 0 \\
\hline 0 & 0 & 0 & 0 & 0 & 0 & 0 & 0 & 0 & 0 & 0 & 0 & 0 & 0 \\
\hline 0 & 0 & 0 & 0 & 0 & 0 & 0 & 0 & 0 & 0 & 0 & 0 & 0 & 0 \\
\hline 0 & 0 & 0 & 0 & 0 & 0 & 0 & 0 & 0 & 0 & 0 & 0 & 0 & 0 \\
\hline 0 & 0 & 0 & 0 & 1 & 0 & 0 & 0 & 0 & 0 & 0 & 0 & 0 & 1 \\
\hline 0 & 0 & 0 & 0 & 0 & 0 & 0 & 0 & 0 & 0 & 0 & 0 & 0 & 0 \\
\hline 0 & 0 & 0 & 0 & 0 & 0 & 0 & 0 & 0 & 0 & 0 & 0 & 0 & 0 \\
\hline 0 & 0 & 0 & 0 & 0 & 0 & 0 & 0 & 0 & 0 & 0 & 0 & 0 & 0 \\
\hline 0 & 0 & 0 & 0 & 0 & 0 & 0 & 0 & 0 & 0 & 0 & 0 & 0 & 0 \\
\hline 0 & 0 & 0 & 0 & 0 & 0 & 0 & 0 & 0 & 0 & 0 & 0 & 0 & 0 \\
\hline 0 & 0 & 0 & 0 & 0 & 0 & 0 & 0 & 0 & 0 & 0 & 0 & 0 & 0 \\
\hline 0 & 0 & 0 & 0 & 0 & 0 & 0 & 0 & 0 & 0 & 0 & 0 & 0 & 0 \\
\hline 0 & 0 & 0 & 0 & 0 & 0 & 0 & 0 & 0 & 0 & 5 & 0 & 0 & 0 \\
\hline 0 & 0 & 0 & 0 & 0 & 0 & 0 & 0 & 0 & 0 & 0 & 0 & 0 & 0 \\
\hline 0 & 0 & 0 & 0 & 0 & 0 & 0 & 0 & 0 & 0 & 0 & 0 & 0 & 0 \\
\hline 0 & 0 & 0 & 0 & 0 & 0 & 0 & 0 & 0 & 0 & 0 & 0 & 0 & 0 \\
\hline 0 & 0 & 0 & 0 & 0 & 0 & 0 & 0 & 0 & 0 & 0 & 0 & 0 & 0 \\
\hline 0 & 0 & 0 & 0 & 0 & 0 & 0 & 0 & 0 & 0 & 0 & 0 & 0 & 0 \\
\hline 0 & 0 & 0 & 0 & 0 & 0 & 0 & 0 & 0 & 0 & 0 & 0 & 0 & 0 \\
\hline 0 & 0 & 0 & 0 & 0 & 0 & 0 & 0 & 0 & 0 & 0 & 0 & 0 & 0 \\
\hline 0 & 0 & 0 & 0 & 0 & 0 & 0 & 0 & 0 & 0 & 0 & 0 & 0 & 0 \\
\hline 0 & 0 & 0 & 0 & 0 & 0 & 0 & 0 & 0 & 0 & 0 & 0 & 0 & 0 \\
\hline
\end{tabular}

(continúa...) 
$\begin{array}{llllllllllllll}\text { P16 } & \text { P17 } & \text { P18 } & \text { P19 } & \text { P20 } & \text { P21 } & \text { P22 } & \text { P23 } & \text { P24 } & \text { P25 } & \text { P26 } & \text { P27 } & \text { P28 } & \text { P29 }\end{array}$

Loxopterygium huasango Spruce ex Engl.

Tagetes sp.

Vernonia sp.

Encelia canescens Lam. var. canescens

Tecoma sp.

Cochlospermum vitifolium (Willd.) Spreng.

Cordia lutea Lam.

Bursera graveolens (H. B. K.) Triana \& Planch.

Opuntia sp.

Neoraimondia arequipensis (Meyen) Backeb.

Armatocereus cartwrightianus (Britton \& Rose) Backeb. ex A.W. Hill Capparis sp. 2

Capparis crotonoides Kunth

Capparis mollis Kunth

Capparis avicenniifolia Kunth

Capparis scabrida Kunth

Maytenus sp.2

Maytenus sp.1

Ipomoea carnea Jacquin

Croton sp.

Croton cf. wagneri Müll. Arg.

Hyptis sp.

Senna undulata (Benth.) H.Irwin \& Barneby

Parkinsonia praecox (Ruiz \& Pav.) Hawkins subsp. praecox

Caesalpinia glabrata Kunth

Albizia multiflora (Kunth) Barneby \& J.W. Grimes

Mimosa cf. acantholoba (Humb. \& Bonpl. ex Willd.) Poir.

Leucaena trichodes (Jacq.) Benth.

Pithecellobium excelsum (Kunth) Mart.

Acacia macracantha H. \& B. ex Willdenow

Prosopis pallida (Humb. \& Bonpl. ex Willd.) Kunth

Piscidia carthagenensis Jacq.

Geoffroea spinosa Jacq.

Erythrina smithiana Krukoff

Buddleja americana L.

Ceiba trichistandra (A. Gray) Bakh.

Eriotheca ruizii (K. Schum.) A. Robyns

Guazuma ulmifolia (Lam.) Kuntze var. ulmifolia

Muntingia calabura L.

Ficus nymphacifolia Mill.

Myrcianthes discolor (HBK) McVaugh

Bougainvillea peruviana Bonpl.

Bougainvillea pachyphylla Heimerl ex Standl.

Pisonia macranthocarpa J. D. Smith

Ximenia americana L.

Priogymnanthus sp.

Piper sp.

Coccoloba densifrons Mart.

Coccoloba sp.

Coccoloba ruiziana Lindau

Scutia spicata (Humb. \& Bonpl. ex Willd.) Weberb. var. spicata

Alseis peruviana Standl.

Simira rubescens (Benth.) Bremek. ex Steyerm.

Sideroxylon sp.

Galvesia fruticosa J. F. Gmel.

Duranta sp.

Acnistus arborescens (L.) Schltdl.

Cestrum auriculatum L'Her.

Celtis iguanaea (Jacq.) Sarg.

Phoradendron sp.

\begin{tabular}{|c|c|c|c|c|c|c|c|c|c|c|c|c|c|}
\hline 14 & 0 & 1 & 2 & 1 & 0 & 2 & 1 & 2 & 0 & 0 & 0 & 0 & 1 \\
\hline 0 & 0 & 0 & 0 & 0 & 0 & 0 & 0 & 0 & 0 & 0 & 0 & 0 & 0 \\
\hline 0 & 0 & 0 & 0 & 0 & 0 & 0 & 0 & 0 & 0 & 0 & 0 & 0 & 0 \\
\hline 0 & 0 & 0 & 0 & 0 & 0 & 0 & 0 & 0 & 0 & 0 & 0 & 0 & 5 \\
\hline 0 & 0 & 0 & 0 & 0 & 0 & 0 & 0 & 0 & 0 & 0 & 0 & 0 & 0 \\
\hline 1 & 0 & 0 & 0 & 0 & 0 & 0 & 0 & 0 & 0 & 0 & 0 & 0 & 0 \\
\hline 4 & 0 & 21 & 14 & 8 & 7 & 20 & 33 & 21 & 6 & 12 & 17 & 8 & 9 \\
\hline 1 & 0 & 3 & 2 & 0 & 1 & 0 & 0 & 1 & 0 & 0 & 0 & 0 & 0 \\
\hline 0 & 0 & 0 & 0 & 0 & 0 & 0 & 0 & 0 & 0 & 0 & 0 & 0 & 0 \\
\hline 0 & 0 & 0 & 0 & 0 & 0 & 0 & 0 & 0 & 0 & 0 & 4 & 0 & 0 \\
\hline 0 & 0 & 0 & 0 & 0 & 0 & 0 & 0 & 10 & 0 & 0 & 0 & 0 & 0 \\
\hline 0 & 0 & 0 & 0 & 0 & 0 & 0 & 0 & 0 & 0 & 0 & 0 & 0 & 0 \\
\hline 0 & 0 & 0 & 0 & 0 & 0 & 0 & 0 & 0 & 0 & 0 & 0 & 0 & 0 \\
\hline 0 & 0 & 0 & 2 & 0 & 0 & 0 & 0 & 0 & 0 & 0 & 0 & 0 & 0 \\
\hline 0 & 0 & 0 & 0 & 0 & 0 & 0 & 0 & 1 & 0 & 0 & 0 & 0 & 4 \\
\hline 0 & 0 & 0 & 0 & 0 & 0 & 0 & 1 & 1 & 0 & 0 & 0 & 0 & 0 \\
\hline 0 & 0 & 0 & 0 & 0 & 0 & 0 & 0 & 0 & 0 & 0 & 0 & 0 & 0 \\
\hline 0 & 0 & 0 & 0 & 0 & 0 & 0 & 0 & 0 & 0 & 0 & 0 & 0 & 0 \\
\hline 0 & 0 & 0 & 0 & 0 & 0 & 0 & 0 & 0 & 0 & 0 & 0 & 0 & 0 \\
\hline 0 & 0 & 0 & 0 & 0 & 0 & 0 & 0 & 0 & 0 & 0 & 0 & 0 & 0 \\
\hline 0 & 0 & 0 & 0 & 0 & 0 & 0 & 0 & 0 & 0 & 0 & 0 & 0 & 0 \\
\hline 0 & 0 & 0 & 0 & 0 & 0 & 0 & 0 & 0 & 0 & 0 & 0 & 0 & 0 \\
\hline 0 & 0 & 0 & 0 & 0 & 0 & 0 & 0 & 0 & 0 & 0 & 0 & 0 & 0 \\
\hline 0 & 0 & 0 & 0 & 0 & 0 & 0 & 0 & 2 & 0 & 0 & 0 & 1 & 0 \\
\hline 0 & 1 & 8 & 1 & 13 & 1 & 1 & 1 & 0 & 0 & 0 & 0 & 0 & 0 \\
\hline 1 & 0 & 0 & 0 & 0 & 0 & 0 & 0 & 0 & 0 & 0 & 0 & 0 & 0 \\
\hline 1 & 0 & 0 & 0 & 0 & 0 & 0 & 0 & 0 & 0 & 0 & 0 & 0 & 0 \\
\hline 0 & 0 & 0 & 0 & 0 & 0 & 0 & 0 & 0 & 0 & 0 & 0 & 0 & 0 \\
\hline 1 & 14 & 1 & 0 & 0 & 0 & 0 & 0 & 0 & 0 & 0 & 0 & 0 & 0 \\
\hline 0 & 0 & 0 & 0 & 0 & 0 & 1 & 0 & 12 & 0 & 0 & 0 & 0 & 11 \\
\hline 0 & 0 & 3 & 0 & 0 & 4 & 9 & 20 & 55 & 46 & 100 & 54 & 57 & 16 \\
\hline 0 & 1 & 0 & 0 & 0 & 0 & 0 & 0 & 0 & 0 & 0 & 0 & 0 & 0 \\
\hline 0 & 2 & 0 & 0 & 0 & 0 & 0 & 0 & 0 & 0 & 0 & 0 & 0 & 0 \\
\hline 7 & 0 & 0 & 0 & 0 & 0 & 0 & 0 & 0 & 0 & 0 & 0 & 0 & 0 \\
\hline 0 & 0 & 0 & 0 & 0 & 0 & 0 & 0 & 0 & 0 & 0 & 0 & 0 & 0 \\
\hline 0 & 0 & 0 & 0 & 0 & 0 & 0 & 0 & 0 & 0 & 0 & 0 & 0 & 0 \\
\hline 1 & 4 & 0 & 1 & 0 & 0 & 0 & 0 & 0 & 0 & 0 & 0 & 0 & 0 \\
\hline 0 & 0 & 0 & 0 & 0 & 0 & 4 & 0 & 12 & 0 & 0 & 0 & 0 & 0 \\
\hline 0 & 0 & 0 & 0 & 0 & 0 & 0 & 0 & 0 & 0 & 0 & 0 & 0 & 0 \\
\hline 0 & 0 & 0 & 0 & 0 & 0 & 0 & 0 & 0 & 0 & 0 & 0 & 0 & 0 \\
\hline 0 & 0 & 0 & 0 & 0 & 0 & 0 & 0 & 0 & 0 & 0 & 0 & 0 & 0 \\
\hline 0 & 0 & 0 & 0 & 0 & 0 & 0 & 0 & 0 & 0 & 0 & 0 & 0 & 0 \\
\hline 1 & 1 & 1 & 0 & 0 & 0 & 0 & 0 & 1 & 0 & 0 & 0 & 0 & 0 \\
\hline 0 & 0 & 0 & 0 & 0 & 0 & 0 & 0 & 0 & 0 & 0 & 0 & 0 & 0 \\
\hline 0 & 0 & 0 & 0 & 0 & 0 & 0 & 0 & 0 & 0 & 0 & 0 & 0 & 0 \\
\hline 0 & 0 & 0 & 0 & 0 & 0 & 0 & 0 & 0 & 0 & 0 & 0 & 0 & 0 \\
\hline 0 & 0 & 0 & 0 & 0 & 0 & 0 & 0 & 0 & 0 & 0 & 0 & 0 & 0 \\
\hline 0 & 0 & 0 & 0 & 0 & 0 & 0 & 0 & 0 & 0 & 0 & 0 & 0 & 0 \\
\hline 0 & 0 & 0 & 0 & 0 & 0 & 0 & 0 & 0 & 0 & 0 & 0 & 0 & 0 \\
\hline 0 & 0 & 0 & 11 & 1 & 0 & 0 & 0 & 0 & 0 & 0 & 0 & 0 & 0 \\
\hline 0 & 0 & 0 & 0 & 0 & 0 & 0 & 0 & 0 & 0 & 0 & 0 & 0 & 0 \\
\hline 7 & 1 & 0 & 0 & 0 & 0 & 0 & 0 & 0 & 0 & 0 & 0 & 0 & 0 \\
\hline 3 & 11 & 0 & 0 & 0 & 0 & 0 & 0 & 0 & 0 & 0 & 0 & 0 & 0 \\
\hline 0 & 0 & 0 & 0 & 0 & 0 & 0 & 0 & 0 & 0 & 0 & 0 & 0 & 0 \\
\hline 0 & 0 & 0 & 0 & 0 & 0 & 0 & 0 & 0 & 0 & 0 & 0 & 0 & 0 \\
\hline 0 & 0 & 0 & 0 & 0 & 0 & 0 & 0 & 0 & 0 & 0 & 0 & 0 & 0 \\
\hline 0 & 0 & 0 & 0 & 0 & 0 & 0 & 0 & 0 & 0 & 0 & 0 & 0 & 0 \\
\hline 0 & 0 & 0 & 0 & 0 & 0 & 0 & 0 & 0 & 0 & 0 & 0 & 0 & 0 \\
\hline 0 & 0 & 0 & 0 & 0 & 0 & 0 & 0 & 0 & 0 & 0 & 0 & 0 & 0 \\
\hline 0 & 0 & 0 & 0 & 0 & 0 & 0 & 0 & 0 & 0 & 0 & 0 & 0 & 0 \\
\hline
\end{tabular}


Anexo 2. (continuación)

\section{Especie}

$\begin{array}{llllllllllllll}\text { P30 } & \text { P31 } & \text { P32 } & \text { P33 } & \text { P34 } & \text { P35 } & \text { P36 } & \text { P37 } & \text { P38 } & \text { P39 } & \text { P40 } & \text { P41 } & \text { P42 } & \text { P43 }\end{array}$

Loxopterygium huasango Spruce ex Engl.

Tagetes sp.

Vernonia sp.

Encelia canescens Lam. var. canescens

Tecoma sp.

Cochlospermum vitifolium (Willd.) Spreng

Cordia lutea Lam.

Bursera graveolens (H. B. K.) Triana \& Planch.

Opuntia sp.

Neoraimondia arequipensis (Meyen) Backeb.

Armatocereus cartwrightianus (Britton \& Rose) Backeb. ex A.W.Hill

Capparis sp. 2

Capparis crotonoides Kunth

Capparis mollis Kunth

Capparis avicenniifolia Kunth

Capparis scabrida Kunth

Maytenus sp. 2

Maytenus sp.1

Ipomoea carnea Jacquin

Croton sp.

Croton cf. wagneri Müll. Arg.

Hyptis sp.

Senna undulata (Benth.) H.Irwin \& Barneby

Parkinsonia praecox (Ruiz \& Pav.) Hawkins subsp. praecox

Caesalpinia glabrata Kunth

Albizia multiflora (Kunth) Barneby \& J.W. Grimes

Mimosa cf. acantholoba (Humb. \& Bonpl. ex Willd.) Poir.

Leucaena trichodes (Jacq.) Benth.

Pithecellobium excelsum (Kunth) Mart.

Acacia macracantha H. \& B. ex Willdenow

Prosopis pallida (Humb. \& Bonpl. ex Willd.) Kunth

Piscidia carthagenensis Jacq.

Geoffroea spinosa Jacq.

Erythrina smithiana Krukoff

Buddleja americana $\mathrm{L}$.

Ceiba trichistandra (A. Gray) Bakh.

Eriotheca ruizii (K. Schum.) A. Robyns

Guazuma ulmifolia (Lam.) Kuntze var. ulmifolia

Muntingia calabura L.

Ficus nymphacifolia Mill.

Myrcianthes discolor (HBK) McVaugh

Bougainvillea peruviana Bonpl.

Bougainvillea pachyphylla Heimerl ex Standl.

Pisonia macranthocarpa J. D. Smith

Ximenia americana $\mathrm{L}$.

Priogymnanthus sp.

Piper sp.

Coccoloba densifrons Mart.

Coccoloba sp.

Coccoloba ruiziana Lindau

Scutia spicata (Humb. \& Bonpl. ex Willd.) Weberb. var. spicata

Alseis peruviana Standl.

Simira rubescens (Benth.) Bremek. ex Steyerm.

Sideroxylon $\mathrm{sp}$.

Galvesia fruticosa J. F. Gmel.

Duranta sp.

Acnistus arborescens (L.) Schltdl.

Cestrum auriculatum L'Her.

Celtis iguanaea (Jacq.) Sarg.

Phoradendron sp.

\begin{tabular}{|c|c|c|c|c|c|c|c|c|c|c|c|c|c|}
\hline 0 & 34 & 0 & 0 & 0 & 0 & 0 & 1 & 7 & 1 & 3 & 2 & 2 & 5 \\
\hline 0 & 0 & 0 & 0 & 0 & 0 & 1 & 0 & 0 & 0 & 2 & 0 & 0 & 0 \\
\hline 0 & 0 & 0 & 0 & 0 & 0 & 0 & 0 & 0 & 0 & 0 & 0 & 0 & 0 \\
\hline 15 & 31 & 2 & 0 & 0 & 16 & 0 & 0 & 0 & 0 & 0 & 0 & 0 & 0 \\
\hline 0 & 0 & 0 & 0 & 0 & 0 & 0 & 0 & 0 & 0 & 0 & 0 & 0 & 0 \\
\hline 0 & 0 & 0 & 0 & 0 & 0 & 0 & 0 & 0 & 0 & 0 & 0 & 0 & 0 \\
\hline 0 & 16 & 7 & 0 & 0 & 0 & 28 & 11 & 4 & 45 & 20 & 21 & 9 & 6 \\
\hline 0 & 2 & 0 & 0 & 0 & 0 & 0 & 5 & 2 & 0 & 2 & 0 & 0 & 1 \\
\hline 0 & 0 & 0 & 0 & 0 & 0 & 0 & 0 & 0 & 0 & 0 & 0 & 0 & 0 \\
\hline 0 & 0 & 0 & 0 & 0 & 0 & 0 & 0 & 0 & 0 & 1 & 0 & 1 & 0 \\
\hline 0 & 0 & 0 & 0 & 0 & 0 & 7 & 0 & 0 & 7 & 0 & 2 & 0 & 0 \\
\hline 0 & 0 & 0 & 0 & 0 & 0 & 0 & 0 & 0 & 0 & 0 & 0 & 0 & 0 \\
\hline 0 & 5 & 1 & 0 & 0 & 0 & 0 & 0 & 2 & 0 & 2 & 3 & 8 & 0 \\
\hline 0 & 0 & 0 & 0 & 0 & 0 & 0 & 0 & 0 & 1 & 0 & 0 & 0 & 0 \\
\hline 2 & 0 & 1 & 8 & 1 & 1 & 0 & 0 & 0 & 0 & 0 & 0 & 0 & 0 \\
\hline 0 & 1 & 3 & 1 & 3 & 5 & 0 & 3 & 6 & 0 & 2 & 6 & 6 & 1 \\
\hline 0 & 0 & 0 & 0 & 0 & 0 & 0 & 0 & 0 & 0 & 0 & 0 & 0 & 0 \\
\hline 0 & 0 & 0 & 0 & 0 & 0 & 0 & 0 & 0 & 0 & 0 & 0 & 0 & 0 \\
\hline 0 & 0 & 0 & 0 & 0 & 0 & 0 & 0 & 0 & 2 & 0 & 0 & 1 & 0 \\
\hline 0 & 0 & 0 & 0 & 0 & 0 & 0 & 0 & 0 & 0 & 0 & 0 & 0 & 0 \\
\hline 0 & 0 & 0 & 0 & 0 & 0 & 0 & 0 & 0 & 0 & 0 & 0 & 0 & 0 \\
\hline 0 & 0 & 0 & 0 & 0 & 0 & 0 & 0 & 0 & 0 & 0 & 0 & 0 & 0 \\
\hline 0 & 0 & 0 & 0 & 0 & 0 & 0 & 0 & 0 & 0 & 0 & 0 & 0 & 0 \\
\hline 0 & 1 & 0 & 0 & 0 & 0 & 0 & 0 & 0 & 0 & 0 & 0 & 0 & 0 \\
\hline 0 & 0 & 0 & 0 & 0 & 0 & 0 & 3 & 1 & 6 & 1 & 0 & 0 & 0 \\
\hline 0 & 0 & 0 & 0 & 0 & 0 & 0 & 0 & 0 & 0 & 0 & 0 & 0 & 0 \\
\hline 0 & 0 & 0 & 0 & 0 & 0 & 5 & 23 & 30 & 4 & 0 & 2 & 0 & 0 \\
\hline 0 & 0 & 0 & 0 & 0 & 0 & 0 & 0 & 0 & 0 & 0 & 0 & 0 & 0 \\
\hline 0 & 0 & 0 & 0 & 0 & 0 & 0 & 0 & 0 & 0 & 0 & 0 & 0 & 0 \\
\hline 162 & 15 & 13 & 0 & 1 & 42 & 12 & 0 & 0 & 0 & 0 & 0 & 0 & 0 \\
\hline 30 & 6 & 1 & 3 & 1 & 9 & 2 & 0 & 0 & 0 & 0 & 0 & 0 & 0 \\
\hline 0 & 0 & 0 & 0 & 0 & 0 & 0 & 0 & 0 & 0 & 0 & 0 & 0 & 0 \\
\hline 0 & 0 & 0 & 0 & 0 & 0 & 0 & 0 & 0 & 0 & 0 & 0 & 0 & 0 \\
\hline 0 & 0 & 0 & 0 & 0 & 0 & 0 & 0 & 0 & 0 & 0 & 0 & 0 & 0 \\
\hline 0 & 0 & 0 & 0 & 0 & 0 & 0 & 0 & 0 & 0 & 0 & 0 & 0 & 0 \\
\hline 0 & 0 & 0 & 0 & 0 & 0 & 2 & 3 & 0 & 0 & 0 & 0 & 0 & 0 \\
\hline 0 & 0 & 0 & 0 & 0 & 0 & 0 & 5 & 10 & 0 & 0 & 0 & 0 & 0 \\
\hline 0 & 4 & 1 & 0 & 0 & 0 & 0 & 0 & 2 & 1 & 2 & 1 & 1 & 7 \\
\hline 0 & 0 & 0 & 0 & 0 & 0 & 0 & 0 & 0 & 0 & 0 & 0 & 0 & 0 \\
\hline 0 & 0 & 0 & 0 & 0 & 0 & 0 & 0 & 0 & 0 & 0 & 0 & 0 & 0 \\
\hline 0 & 0 & 0 & 0 & 0 & 0 & 0 & 0 & 0 & 0 & 0 & 0 & 0 & 0 \\
\hline 0 & 0 & 0 & 0 & 0 & 0 & 0 & 0 & 0 & 0 & 0 & 0 & 0 & 0 \\
\hline 0 & 0 & 0 & 0 & 0 & 0 & 1 & 0 & 1 & 0 & 0 & 0 & 0 & 0 \\
\hline 0 & 0 & 0 & 0 & 0 & 0 & 0 & 0 & 0 & 0 & 0 & 0 & 0 & 0 \\
\hline 0 & 2 & 0 & 0 & 0 & 0 & 0 & 2 & 0 & 0 & 0 & 1 & 0 & 0 \\
\hline 0 & 0 & 0 & 0 & 0 & 0 & 0 & 0 & 0 & 0 & 0 & 0 & 0 & 0 \\
\hline 0 & 0 & 0 & 0 & 0 & 0 & 0 & 0 & 0 & 0 & 0 & 0 & 0 & 0 \\
\hline 0 & 0 & 0 & 0 & 0 & 0 & 0 & 0 & 0 & 0 & 0 & 0 & 0 & 0 \\
\hline 0 & 0 & 0 & 0 & 0 & 0 & 0 & 0 & 0 & 0 & 0 & 0 & 0 & 0 \\
\hline 0 & 0 & 0 & 0 & 0 & 0 & 0 & 0 & 0 & 0 & 0 & 0 & 0 & 0 \\
\hline 0 & 1 & 0 & 0 & 0 & 0 & 0 & 0 & 0 & 0 & 0 & 0 & 0 & 0 \\
\hline 0 & 0 & 0 & 0 & 0 & 0 & 0 & 0 & 0 & 0 & 0 & 0 & 0 & 0 \\
\hline 0 & 0 & 0 & 0 & 0 & 0 & 0 & 0 & 0 & 0 & 0 & 0 & 0 & 0 \\
\hline 0 & 0 & 0 & 0 & 0 & 0 & 0 & 0 & 0 & 0 & 0 & 0 & 0 & 0 \\
\hline 0 & 0 & 0 & 0 & 0 & 0 & 0 & 0 & 0 & 0 & 0 & 1 & 0 & 1 \\
\hline 0 & 0 & 0 & 0 & 0 & 0 & 0 & 0 & 0 & 0 & 0 & 0 & 0 & 0 \\
\hline 0 & 0 & 0 & 0 & 0 & 0 & 0 & 0 & 0 & 0 & 0 & 0 & 0 & 0 \\
\hline 0 & 0 & 0 & 0 & 0 & 0 & 0 & 0 & 0 & 0 & 0 & 0 & 0 & 0 \\
\hline 0 & 0 & 0 & 0 & 0 & 0 & 0 & 0 & 0 & 0 & 0 & 0 & 0 & 0 \\
\hline 0 & 0 & 0 & 0 & 0 & 0 & 0 & 0 & 0 & 0 & 0 & 0 & 0 & 0 \\
\hline
\end{tabular}

(continúa...) 


\begin{tabular}{|c|c|c|c|c|c|c|c|c|c|c|c|c|c|c|}
\hline Especie & P44 & P45 & P46 & $\mathbf{P} 47$ & $\mathbf{P} 48$ & P49 & P50 & P51 & P52 & P53 & P54 & P55 & P56 & P57 \\
\hline Loxopterygium huasango Spruce ex Engl. & 0 & 0 & 1 & 1 & 0 & 1 & 2 & 0 & 3 & 2 & 1 & 1 & 0 & 0 \\
\hline Tagetes sp. & 0 & 0 & 0 & 0 & 0 & 0 & 0 & 0 & 0 & 0 & 0 & 0 & 0 & 0 \\
\hline Vernonia sp. & 0 & 0 & 0 & 0 & 0 & 0 & 0 & 0 & 0 & 0 & 0 & 0 & 0 & 0 \\
\hline Encelia canescens Lam. var. canescens & 1 & 0 & 0 & 0 & 0 & 0 & 0 & 0 & 0 & 0 & 0 & 0 & 0 & 0 \\
\hline Tecoma sp. & 0 & 0 & 0 & 0 & 0 & 0 & 0 & 0 & 0 & 0 & 0 & 0 & 0 & 0 \\
\hline Cochlospermum vitifolium (Willd.) Spreng. & 0 & 0 & 1 & 0 & 0 & 0 & 0 & 0 & 0 & 0 & 0 & 5 & 0 & 0 \\
\hline Cordia lutea Lam. & 0 & 17 & 16 & 8 & 10 & 4 & 23 & 55 & 0 & 25 & 42 & 33 & 0 & 0 \\
\hline Bursera graveolens (H. B. K.) Triana \& Planch. & 0 & 0 & 3 & 1 & 0 & 0 & 1 & 0 & 1 & 0 & 1 & 0 & 0 & 0 \\
\hline Opuntia sp. & 0 & 0 & 0 & 1 & 1 & 0 & 0 & 0 & 0 & 0 & 0 & 0 & 0 & 0 \\
\hline Neoraimondia arequipensis (Meyen) Backeb. & 0 & 0 & 0 & 0 & 0 & 0 & 0 & 4 & 0 & 0 & 1 & 0 & 0 & 0 \\
\hline Armatocereus cartwrightianus (Britton \& Rose) Backeb. ex A.W. Hill & 0 & 0 & 18 & 1 & 1 & 0 & 0 & 0 & 0 & 0 & 1 & 4 & 0 & 0 \\
\hline Capparis sp. 2 & 0 & 0 & 0 & 0 & 0 & 0 & 0 & 0 & 0 & 0 & 0 & 0 & 0 & 0 \\
\hline Capparis crotonoides Kunth & 0 & 0 & 0 & 0 & 0 & 0 & 0 & 0 & 0 & 0 & 0 & 0 & 0 & 0 \\
\hline Capparis mollis Kunth & 0 & 0 & 0 & 0 & 0 & 0 & 0 & 0 & 0 & 0 & 0 & 0 & 0 & 0 \\
\hline Capparis avicenniifolia Kunth & 0 & 0 & 0 & 0 & 0 & 0 & 0 & 0 & 0 & 0 & 0 & 0 & 0 & 0 \\
\hline Capparis scabrida Kunth & 12 & 0 & 0 & 1 & 0 & 0 & 0 & 0 & 0 & 0 & 0 & 0 & 0 & 4 \\
\hline Maytenus sp.2 & 0 & 0 & 0 & 0 & 0 & 0 & 0 & 0 & 0 & 0 & 0 & 0 & 0 & 0 \\
\hline Maytenus sp.1 & 0 & 0 & 0 & 0 & 0 & 0 & 0 & 0 & 0 & 0 & 0 & 0 & 0 & 0 \\
\hline Ipomoea carnea Jacquin & 0 & 0 & 14 & 1 & 135 & 23 & 9 & 21 & 4 & 9 & 57 & 0 & 0 & 0 \\
\hline Croton sp. & 0 & 0 & 0 & 0 & 0 & 0 & 0 & 0 & 0 & 0 & 0 & 0 & 0 & 0 \\
\hline Croton cf. wagneri Müll. Arg. & 0 & 0 & 31 & 1 & 53 & 255 & 0 & 0 & 0 & 0 & 0 & 0 & 0 & 0 \\
\hline Hyptis sp. & 0 & 0 & 0 & 0 & 0 & 0 & 0 & 0 & 0 & 0 & 0 & 0 & 0 & 0 \\
\hline Senna undulata (Benth.) H.Irwin \& Barneby & 0 & 0 & 0 & 0 & 0 & 0 & 0 & 0 & 0 & 0 & 0 & 0 & 0 & 0 \\
\hline Parkinsonia praecox (Ruiz \& Pav.) Hawkins subsp. praecox & 21 & 10 & 0 & 0 & 0 & 0 & 0 & 0 & 0 & 0 & 0 & 0 & 0 & 0 \\
\hline Caesalpinia glabrata Kunth & 0 & 0 & 0 & 1 & 2 & 3 & 2 & 2 & 0 & 0 & 0 & 0 & 0 & 0 \\
\hline Albizia multiflora (Kunth) Barneby \& J.W. Grimes & 0 & 0 & 0 & 0 & 0 & 1 & 0 & 0 & 0 & 0 & 0 & 0 & 0 & 0 \\
\hline Mimosa cf. acantholoba (Humb. \& Bonpl. ex Willd.) Poir. & 0 & 0 & 2 & 1 & 0 & 0 & 0 & 0 & 0 & 0 & 0 & 0 & 0 & 0 \\
\hline Leucaena trichodes (Jacq.) Benth. & 0 & 0 & 0 & 0 & 0 & 0 & 0 & 0 & 0 & 0 & 0 & 0 & 0 & 0 \\
\hline Pithecellobium excelsum (Kunth) Mart. & 0 & 0 & 0 & 0 & 0 & 5 & 0 & 0 & 0 & 3 & 0 & 1 & 0 & 0 \\
\hline Acacia macracantha H. \& B. ex Willdenow & 0 & 0 & 1 & 0 & 0 & 0 & 0 & 0 & 0 & 0 & 0 & 0 & 0 & 0 \\
\hline Prosopis pallida (Humb. \& Bonpl. ex Willd.) Kunth & 39 & 49 & 0 & 0 & 0 & 0 & 0 & 85 & 0 & 0 & 5 & 0 & 12 & 3 \\
\hline Piscidia carthagenensis Jacq. & 0 & 0 & 0 & 0 & 0 & 2 & 0 & 0 & 17 & 2 & 0 & 0 & 0 & 0 \\
\hline Geoffroea spinosa Jacq. & 0 & 0 & 0 & 0 & 0 & 0 & 0 & 0 & 1 & 0 & 0 & 0 & 0 & 0 \\
\hline Erythrina smithiana Krukoff & 0 & 0 & 0 & 0 & 0 & 0 & 0 & 0 & 1 & 0 & 0 & 0 & 0 & 0 \\
\hline Buddleja americana $\mathrm{L}$. & 0 & 0 & 0 & 0 & 0 & 0 & 0 & 0 & 0 & 0 & 0 & 0 & 0 & 0 \\
\hline Ceiba trichistandra (A. Gray) Bakh. & 0 & 0 & 1 & 1 & 0 & 2 & 0 & 0 & 1 & 2 & 0 & 0 & 0 & 0 \\
\hline Eriotheca ruizii (K. Schum.) A. Robyns & 0 & 0 & 1 & 1 & 0 & 0 & 0 & 0 & 4 & 0 & 0 & 0 & 0 & 0 \\
\hline Guazuma ulmifolia (Lam.) Kuntze var. ulmifolia & 0 & 0 & 2 & 2 & 0 & 0 & 0 & 0 & 5 & 1 & 0 & 81 & 0 & 0 \\
\hline Muntingia calabura L. & 0 & 0 & 0 & 0 & 0 & 0 & 0 & 0 & 0 & 0 & 0 & 0 & 0 & 0 \\
\hline Ficus nymphacifolia Mill. & 0 & 0 & 0 & 0 & 0 & 0 & 0 & 0 & 0 & 0 & 0 & 0 & 0 & 0 \\
\hline Myrcianthes discolor (HBK) McVaugh & 0 & 0 & 0 & 0 & 0 & 0 & 0 & 0 & 0 & 0 & 0 & 0 & 0 & 0 \\
\hline Bougainvillea peruviana Bonpl. & 0 & 0 & 0 & 0 & 0 & 0 & 0 & 0 & 0 & 0 & 0 & 0 & 0 & 0 \\
\hline Bougainvillea pachyphylla Heimerl ex Standl. & 0 & 0 & 6 & 3 & 2 & 1 & 0 & 0 & 1 & 1 & 1 & 9 & 0 & 0 \\
\hline Pisonia macranthocarpa J. D. Smith & 0 & 0 & 0 & 0 & 0 & 0 & 0 & 0 & 0 & 0 & 0 & 0 & 0 & 0 \\
\hline Ximenia americana L. & 0 & 0 & 1 & 1 & 0 & 0 & 0 & 0 & 1 & 0 & 0 & 0 & 0 & 0 \\
\hline Priogymnanthus sp. & 0 & 0 & 0 & 0 & 0 & 0 & 0 & 0 & 0 & 0 & 0 & 0 & 0 & 0 \\
\hline Piper sp. & 0 & 0 & 0 & 0 & 0 & 0 & 0 & 0 & 0 & 0 & 0 & 0 & 0 & 0 \\
\hline Coccoloba densifrons Mart. & 0 & 0 & 0 & 0 & 0 & 0 & 0 & 0 & 0 & 0 & 0 & 0 & 0 & 0 \\
\hline Coccoloba sp. & 0 & 0 & 0 & 0 & 0 & 0 & 0 & 0 & 0 & 0 & 0 & 0 & 0 & 0 \\
\hline Coccoloba ruiziana Lindau & 0 & 0 & 0 & 5 & 8 & 4 & 7 & 0 & 7 & 2 & 0 & 5 & 0 & 0 \\
\hline Scutia spicata (Humb. \& Bonpl. ex Willd.) Weberb. var. spicata & 0 & 0 & 0 & 0 & 0 & 0 & 0 & 0 & 0 & 0 & 0 & 0 & 0 & 0 \\
\hline Alseis peruviana Standl. & 0 & 0 & 0 & 0 & 0 & 2 & 0 & 0 & 9 & 4 & 0 & 0 & 0 & 0 \\
\hline Simira rubescens (Benth.) Bremek. ex Steyerm. & 0 & 0 & 0 & 0 & 0 & 0 & 0 & 0 & 46 & 0 & 0 & 0 & 0 & 0 \\
\hline Sideroxylon sp. & 0 & 0 & 0 & 0 & 0 & 0 & 0 & 0 & 0 & 0 & 0 & 0 & 0 & 0 \\
\hline Galvesia fruticosa J. F. Gmel. & 1 & 1 & 0 & 0 & 0 & 0 & 0 & 0 & 0 & 0 & 0 & 0 & 0 & 0 \\
\hline Duranta sp. & 0 & 0 & 0 & 0 & 0 & 0 & 0 & 0 & 0 & 0 & 0 & 0 & 0 & 0 \\
\hline Acnistus arborescens (L.) Schltdl. & 0 & 0 & 0 & 0 & 0 & 0 & 0 & 0 & 0 & 0 & 0 & 0 & 0 & 0 \\
\hline Cestrum auriculatum L'Her. & 0 & 0 & 0 & 0 & 0 & 0 & 0 & 0 & 0 & 0 & 0 & 0 & 0 & 0 \\
\hline Celtis iguanaea (Jacq.) Sarg. & 0 & 0 & 0 & 0 & 0 & 0 & 0 & 0 & 0 & 0 & 0 & 0 & 0 & 0 \\
\hline Phoradendron sp. & 0 & 0 & 0 & 0 & 0 & 0 & 0 & 0 & 0 & 0 & 0 & 0 & 0 & 0 \\
\hline
\end{tabular}


Anexo 2. (continuación)

\begin{tabular}{llllllllll}
\hline Especie & P58 & P59 & P60 & P61 & P62 & P63 & P64 & P65 & P66
\end{tabular}

Loxopterygium huasango Spruce ex Engl.

Tagetes sp.

Vernonia sp.

Encelia canescens Lam. var. canescens

Tecoma sp.

Cochlospermum vitifolium (Willd.) Spreng.

Cordia lutea Lam.

Bursera graveolens (H. B. K.) Triana \& Planch.

Opuntia sp.

Neoraimondia arequipensis (Meyen) Backeb.

Armatocereus cartwrightianus (Britton \& Rose) Backeb. ex A.W.Hill

Capparis sp. 2

Capparis crotonoides Kunth

Capparis mollis Kunth

Capparis avicenniifolia Kunth

Capparis scabrida Kunth

Maytenus sp. 2

Maytenus sp.1

Ipomoea carnea Jacquin

Croton sp.

Croton cf. wagneri Müll. Arg.

Hyptis sp.

Senna undulata (Benth.) H.Irwin \& Barneby

Parkinsonia praecox (Ruiz \& Pav.) Hawkins subsp. praecox

Caesalpinia glabrata Kunth

Albizia multiflora (Kunth) Barneby \& J.W. Grimes

Mimosa cf. acantholoba (Humb. \& Bonpl. ex Willd.) Poir.

Leucaena trichodes (Jacq.) Benth.

Pithecellobium excelsum (Kunth) Mart.

Acacia macracantha H. \& B. ex Willdenow

Prosopis pallida (Humb. \& Bonpl. ex Willd.) Kunth

Piscidia carthagenensis Jacq.

Geoffroea spinosa Jacq.

Erythrina smithiana Krukoff

Buddleja americana $\mathrm{L}$.

Ceiba trichistandra (A. Gray) Bakh.

Eriotheca ruizii (K. Schum.) A. Robyns

Guazuma ulmifolia (Lam.) Kuntze var. ulmifolia

Muntingia calabura L.

Ficus nymphacifolia Mill.

Myrcianthes discolor (HBK) McVaugh

Bougainvillea peruviana Bonpl.

Bougainvillea pachyphylla Heimerl ex Standl.

Pisonia macranthocarpa J. D. Smith

Ximenia americana $\mathrm{L}$.

Priogymnanthus sp.

Piper sp.

Coccoloba densifrons Mart.

Coccoloba sp.

Coccoloba ruiziana Lindau

Scutia spicata (Humb. \& Bonpl. ex Willd.) Weberb. var. spicata

Alseis peruviana Standl.

Simira rubescens (Benth.) Bremek. ex Steyerm.

Sideroxylon sp.

Galvesia fruticosa J. F. Gmel.

Duranta sp.

Acnistus arborescens (L.) Schltdl.

Cestrum auriculatum L'Her.

Celtis iguanaea (Jacq.) Sarg.

Phoradendron sp.

\begin{tabular}{|c|c|c|c|c|c|c|c|}
\hline 0 & 8 & 8 & 4 & 5 & 9 & 3 & 12 \\
\hline 0 & 0 & 0 & 0 & 0 & 0 & 0 & 0 \\
\hline 0 & 10 & 11 & 9 & 8 & 0 & 0 & 1 \\
\hline 1 & 0 & 0 & 0 & 0 & 0 & 0 & 0 \\
\hline 0 & 0 & 0 & 0 & 5 & 0 & 11 & 2 \\
\hline 0 & 0 & 0 & 4 & 2 & 0 & 0 & 0 \\
\hline 0 & 3 & 3 & 1 & 1 & 13 & 0 & 5 \\
\hline 0 & 8 & 6 & 3 & 9 & 7 & 6 & 6 \\
\hline 0 & 0 & 0 & 0 & 0 & 0 & 0 & 0 \\
\hline 0 & 0 & 0 & 0 & 0 & 0 & 0 & 0 \\
\hline 0 & 0 & 0 & 0 & 0 & 0 & 0 & 0 \\
\hline 0 & 0 & 0 & 0 & 0 & 0 & 0 & 0 \\
\hline 0 & 0 & 0 & 0 & 0 & 0 & 0 & 0 \\
\hline 0 & 8 & 8 & 7 & 5 & 6 & 4 & 5 \\
\hline 11 & 0 & 0 & 0 & 0 & 3 & 0 & 0 \\
\hline 1 & 1 & 0 & 5 & 1 & 2 & 0 & 2 \\
\hline 0 & 1 & 3 & 10 & 4 & 0 & 0 & 0 \\
\hline 0 & 12 & 8 & 9 & 10 & 18 & 21 & 1 \\
\hline 0 & 0 & 0 & 0 & 0 & 0 & 0 & 0 \\
\hline 0 & 0 & 0 & 5 & 0 & 0 & 3 & 0 \\
\hline 0 & 0 & 0 & 0 & 0 & 0 & 0 & 0 \\
\hline 0 & 6 & 2 & 6 & 2 & 0 & 0 & 4 \\
\hline 0 & 2 & 1 & 0 & 0 & 0 & 0 & 0 \\
\hline 1 & 0 & 0 & 0 & 0 & 0 & 0 & 0 \\
\hline 0 & 4 & 1 & 3 & 4 & 5 & 3 & 5 \\
\hline 0 & 10 & 7 & 6 & 11 & 11 & 11 & 4 \\
\hline 0 & 0 & 0 & 0 & 0 & 0 & 0 & 0 \\
\hline 0 & 9 & 15 & 12 & 3 & 10 & 17 & 2 \\
\hline 0 & 19 & 20 & 22 & 14 & 24 & 15 & 23 \\
\hline 0 & 10 & 8 & 5 & 10 & 10 & 11 & 4 \\
\hline 5 & 0 & 0 & 0 & 0 & 1 & 0 & 0 \\
\hline 0 & 0 & 0 & 0 & 0 & 0 & 0 & 0 \\
\hline 0 & 3 & 5 & 2 & 1 & 4 & 2 & 3 \\
\hline 0 & 17 & 13 & 16 & 19 & 14 & 18 & 10 \\
\hline 0 & 0 & 11 & 3 & 5 & 0 & 7 & 1 \\
\hline 0 & 0 & 0 & 0 & 0 & 0 & 0 & 2 \\
\hline 0 & 52 & 31 & 46 & 52 & 59 & 44 & 48 \\
\hline 0 & 0 & 0 & 0 & 0 & 0 & 19 & 3 \\
\hline 0 & 6 & 11 & 3 & 10 & 4 & 8 & 6 \\
\hline 0 & 5 & 8 & 4 & 3 & 2 & 10 & 6 \\
\hline 0 & 0 & 0 & 0 & 0 & 0 & 0 & 2 \\
\hline 0 & 1 & 1 & 0 & 1 & 8 & 1 & 0 \\
\hline 0 & 0 & 0 & 0 & 0 & 0 & 0 & 0 \\
\hline 0 & 6 & 9 & 9 & 2 & 0 & 0 & 2 \\
\hline 0 & 0 & 0 & 0 & 0 & 0 & 0 & 0 \\
\hline 0 & 2 & 0 & 12 & 7 & 11 & 20 & 14 \\
\hline 0 & 0 & 0 & 0 & 2 & 0 & 0 & 3 \\
\hline 0 & 0 & 0 & 0 & 3 & 0 & 0 & 8 \\
\hline 0 & 0 & 0 & 10 & 3 & 0 & 0 & 3 \\
\hline 0 & 0 & 0 & 0 & 0 & 0 & 0 & 0 \\
\hline 1 & 3 & 13 & 5 & 3 & 4 & 1 & 5 \\
\hline 0 & 0 & 0 & 0 & 0 & 0 & 0 & 0 \\
\hline 0 & 0 & 0 & 0 & 0 & 0 & 0 & 0 \\
\hline 0 & 1 & 1 & 5 & 0 & 6 & 1 & 1 \\
\hline 0 & 0 & 0 & 0 & 0 & 0 & 0 & 0 \\
\hline 0 & 0 & 0 & 0 & 1 & 2 & 0 & 0 \\
\hline 0 & 0 & 0 & 10 & 7 & 0 & 0 & 0 \\
\hline 0 & 9 & 5 & 6 & 9 & 0 & 1 & 10 \\
\hline 0 & 9 & 5 & 6 & 7 & 20 & 17 & 5 \\
\hline 0 & 0 & 10 & 0 & 0 & 4 & 0 & 1 \\
\hline
\end{tabular}

